\title{
$\beta$ decay of odd- $A$ nuclei with the interacting boson-fermion model based on the Gogny energy density functional
}

\author{
K. Nomura $\odot,{ }^{1, *}$ R. Rodríguez-Guzmán, ${ }^{2}$ and L. M. Robledo ${ }^{3,4}$ \\ ${ }^{1}$ Department of Physics, Faculty of Science, University of Zagreb, HR-10000 Zagreb, Croatia \\ ${ }^{2}$ Physics Department, Kuwait University, 13060 Kuwait, Kuwait \\ ${ }^{3}$ Center for Computational Simulation, Universidad Politńica de Madrid, Campus de Montegancedo, \\ Boadilla del Monte, 28660 Madrid, Spain \\ ${ }^{4}$ Departamento de Física Teórica, Universidad Autónoma de Madrid, E-28049 Madrid, Spain
}

(Received 4 December 2019; accepted 7 February 2020; published 21 February 2020)

\begin{abstract}
The low-energy excitations and $\beta$ decays of odd- $A$ nuclei are studied within the interacting boson-fermion model (IBFM), based on the Gogny-D1M nuclear energy density functional (EDF). The constrained HartreeFock-Bogoliubov (HFB) approximation is employed to compute potential energy surfaces in terms of triaxial quadrupole degrees of freedom for even-even $\mathrm{Xe}$ and $\mathrm{Ba}$ nuclei in the mass $A \approx 130$ region. The mean field approximation also provides spherical single-particle energies and occupation probabilities for the neighboring odd- $A$ nuclei. Those quantities represent a microscopic input for spectroscopic calculations in odd- $A \mathrm{Xe}, \mathrm{Ba}, \mathrm{Cs}$ and La isotopes. The Gamow-Teller (GT) and Fermi (F) transition matrix elements needed to compute $\beta$-decay $\log f t$ values are obtained without any phenomenological fitting. It is shown that both the low-lying states and $\beta$ decays of the studied odd- $A$ systems are described reasonably well within the employed theoretical framework.
\end{abstract}

DOI: 10.1103/PhysRevC.101.024311

\section{INTRODUCTION}

Understanding the structure of the atomic nucleus is essential to accurately model fundamental processes, such as the $\beta$ and $\beta \beta$ decays, and often provides useful insight into other domains of physics; e.g., it has potential impact on the search for new physics beyond the standard model of elementary particles. Experiments have already been performed at major radioactive-ion-beam facilities around the world to measure the $\beta$-decay half-lives of numerous neutron-rich heavy nuclei [1-5]. Those experiments not only are useful for a better understanding of nuclear structure phenomena at extreme neutron-to-proton ratios $N / Z$, but are also instrumental to model the creation of chemical elements in various astrophysical nucleosynthesis scenarios. In addition, $\beta$-decay properties are expected to be sensitive to details of the wave functions of low-lying states of both the parent and daughter nuclei. Therefore, they also serve as a stringent test of various nuclear structure models [6,7]. A number of theoretical calculations have been performed to study $\beta$ decay properties, e.g., in terms of the quasiparticle random phase approximation (QRPA) at various levels of sophistication [8-13], the largescale nuclear shell model [6,14-16], and the interacting boson model [17-24].

The microscopic description of the spectroscopic properties of medium-heavy and heavy nuclei is a highly demanding computational task. In particular, an accurate description of the excitation spectra and transition rates in

\footnotetext{
*knomura@phy.hr
}

odd-mass and/or odd-odd nuclei stills remains a major challenge in today's nuclear structure theory. What complicates the microscopic description of nuclear systems with unpaired nucleons are features such as, for example, the weakening of pairing correlations, the increase of level densities around the Fermi level, polarization effects, and the breaking of time-reversal symmetry in the intrinsic wave functions [25-28]. Spectroscopic studies of odd-mass and/or oddodd nuclei have also been carried out within the framework of the symmetry-projected generator coordinate method (GCM) $[28,29]$. However, from a computational point of view, this kind of approach is very costly if not impossible to apply in heavy nuclei, especially when many valence nucleons are involved and/or multiple shape degrees of freedom need to be taken into account in the symmetry-projected GCM ansatz.

An alternative and numerically feasible EDF-based particle-core coupling approach to the spectroscopy of odd- $A$ nuclei has been developed in previous works [30,31]. Within this approach, the $(\beta, \gamma)$ potential energy surface (PES) of a given even-even core nucleus is computed microscopically using the constrained mean field approximation. The mean field approximation also provides the spherical single-particle energies and occupation numbers for unpaired nucleon(s) in the neighboring odd- $A$ or odd-odd nucleus. Those mean field quantities represent an essential input to build the Hamiltonian of the interacting boson-fermion model (IBFM) [19,32]. Three coupling constants for the boson-fermion interaction terms are determined so as to reproduce reasonably well the experimental low-energy spectrum in a given odd- $A$ system. At the cost of having to determine these few coupling 
constants empirically, the method allows a detailed and simultaneous description of spectroscopy in even-even, odd- $A$, and odd-odd nuclei [33].

It is then interesting to examine whether the IBFM framework based on the microscopic EDF approach can provide at the same time a consistent description of the low-lying states and $\beta$-decay properties in heavy odd- $A$ nuclei. In this work, we study those $\beta$ decays where only Gamow-Teller (GT) and Fermi (F) transitions are involved, that is, the spin of a parent nucleus changes according to $\Delta I=0, \pm 1$ and parity is conserved. The $\beta$ decay $\log f t$ values require the computation of the GT and F transition strengths which can be obtained using the IBFM wave functions for the parent and daughter odd- $A$ nuclei. One of the advantages of our approach is that the IBFM $\beta$ decay calculations do not involve any free parameter associated to the GT and F operators. Therefore, $\beta$ decay properties can be considered a very stringent test for the IBFM wave functions.

In this work, we focus on the $\beta$ decay of odd- $A$ nuclei in the $A \approx 130$ mass region. In Ref. [34], we have already applied the method to describe the $\gamma$-soft-to-near-spherical shape phase transitions in odd $-A \mathrm{Xe}, \mathrm{Cs}, \mathrm{Ba}$, and $\mathrm{La}$ isotopic chains. This mass region is one of the most studied in the nuclear chart in the context of the $\beta$ decay. Moreover, nuclei with $A \approx 130$ exhibit a variety of nuclear structure phenomena such as the relevance of triaxial deformations and the existence of quantum phase transitions from prolate to $\gamma$-soft (or $\mathrm{O}(6)$ limit of IBM [35]) and near spherical shapes as one approaches the $N=82$ neutron shell closure. The evolution of shapes with neutron number might be expected to play a role in the corresponding $\beta$-decay properties. Phenomenological IBFM studies of the low-lying states [36] and $\beta$ decay properties $[21,24]$ have already been performed in the same mass region.

The paper is outlined as follows. In Sec. II, we briefly describe the procedures followed to build the IBFM Hamiltonians from the constrained Gogny-HFB calculations. We use the parametrization D1M [37] of the Gogny-EDF [27,38] because previous studies using the EDF-to-IBM mapping procedure, have shown that the Gogny-D1M EDF provides a reasonable description of the spectroscopic properties in medium-mass, heavy odd- $A$, and odd-odd nuclei $[31,33,34,39]$ in a wide range of nuclei. In the same section, we also introduce the $\beta$-decay operators. The results of our calculations for the low-lying energy levels in the even-even $\mathrm{Xe}$ and $\mathrm{Ba}$ nuclei are discussed in Sec. III. Spectroscopic results from the IBFM calculations for the odd- $A \mathrm{Xe}, \mathrm{Cs}, \mathrm{Ba}$, and $\mathrm{La}$ isotopes are presented in Sec. IV. The $\log f t$ values obtained for the $\beta$ decays of the studied nuclei are discussed in Sec. V. Finally, Sec. VI is devoted to the concluding remarks.

\section{THEORETICAL FRAMEWORK}

\section{A. Hamiltonian}

Let us introduce the IBFM Hamiltonian for odd- $A$ systems. We use the IBMF-2 version of the IBFM (called IBFM-2) and we differentiate between proton and neutron degrees of freedom. In the following, we will simply denote the IBFM-2 as IBFM. The IBFM Hamiltonian reads

$$
\hat{H}=\hat{H}_{\mathrm{B}}+\hat{H}_{\mathrm{F}}^{v}+\hat{H}_{\mathrm{F}}^{\pi}+\hat{H}_{\mathrm{BF}}^{v}+H_{\mathrm{BF}}^{\pi} \cdot
$$

The first term represents the neutron-proton IBM (IBM2) Hamiltonian [40] used to describe the even-even core nucleus:

$$
\hat{H}_{\mathrm{B}}=\epsilon\left(\hat{n}_{d_{\nu}}+\hat{n}_{d_{\pi}}\right)+\kappa \hat{Q}_{\nu} \hat{Q}_{\pi}
$$

Here $\hat{n}_{d_{\rho}}=d_{\rho}^{\dagger} \tilde{d}_{\rho}(\rho=v, \pi)$ is the $d$-boson number operator, and $\hat{Q}_{\rho}=d_{\rho}^{\dagger} s_{\rho}+s_{\rho}^{\dagger} \tilde{d}_{\rho}^{\dagger}+\chi_{\rho}\left(d_{\rho}^{\dagger} \tilde{d}_{\rho}\right)^{(2)}$ is the quadrupole operator. The parameters of the Hamiltonian are denoted by $\epsilon, \kappa, \chi_{\nu}$, and $\chi_{\pi}$. The doubly magic nucleus ${ }^{132} \mathrm{Sn}$ is taken as the inert core for the boson space. The number of neutron $N_{v}$ and proton $N_{\pi}$ bosons equals the number of neutron-hole and proton-particle pairs, respectively [40].

The second and third terms in Eq. (1) represent the Hamiltonians for the odd neutron and the odd proton, respectively. Its generic form is

$$
\hat{H}_{\mathrm{F}}^{\rho}=-\sum_{j_{\rho}} \epsilon_{j_{\rho}} \sqrt{2 j_{\rho}+1}\left(a_{j_{\rho}}^{\dagger} \tilde{a}_{j_{\rho}}\right)^{(0)}
$$

with $\epsilon_{j_{\rho}}$ being the single-particle energy of the odd nucleon. Here, $j_{\rho}$ stands for the angular momentum of the single nucleon. On the other hand, $a_{j_{\rho}}^{(\dagger)}$ and $\tilde{a}_{j_{\rho}}$ represent fermion creation and annihilation operators, with $\tilde{a}_{j m}=(-1)^{j-m} a_{j-m}$. For the fermion valence space, we consider the full neutron and proton major shell $N, Z=50-82$, i.e., $3 s_{1 / 2}, 2 d_{3 / 2}, 2 d_{5 / 2}$, $1 g_{7 / 2}$, and $1 h_{11 / 2}$ orbitals.

The fourth and fifth terms in Eq. (1) represent the coupling of the odd neutron and of the odd proton to the IBM-2 core, respectively:

$$
\hat{H}_{\mathrm{BF}}^{\rho}=\Gamma_{\rho} \hat{Q}_{\rho^{\prime}} \hat{q}_{\rho}+\Lambda_{\rho} \hat{V}_{\rho^{\prime} \rho}+A_{\rho} \hat{n}_{d_{\rho}} \hat{n}_{\rho},
$$

where $\rho^{\prime} \neq \rho$. The first, second, and third terms in the equation above are the quadrupole dynamical, exchange, and monopole terms, respectively. The strength parameters are denoted by $\Gamma_{\rho}, \Lambda_{\rho}$, and $A_{\rho}$. As in the previous studies [41,42], we assume that both the dynamical and exchange terms are dominated by the interaction between unlike particles (i.e., between the odd neutron and proton bosons and between the odd proton and neutron bosons), and that, for the monopole term, the interaction between like particles (i.e., between the odd neutron and neutron bosons and between the odd proton and proton bosons) plays a dominant role. In Eq. (4), $\hat{Q}_{\rho}$ is the same bosonic quadrupole operator as in the IBM-2 Hamiltonian in Eq. (2). The fermionic quadrupole operator $\hat{q}_{\rho}$ reads

$$
\hat{q}_{\rho}=\sum_{j_{\rho} j_{\rho}^{\prime}} \gamma_{j_{\rho} j_{\rho}^{\prime}}\left(a_{j_{\rho}}^{\dagger} \tilde{a}_{j_{\rho}^{\prime}}\right)^{(2)}
$$

where $\quad \gamma_{j_{\rho} j_{\rho}^{\prime}}=\left(u_{j_{\rho}} u_{j_{\rho}^{\prime}}-v_{j_{\rho}} v_{j_{\rho}^{\prime}}\right) Q_{j_{\rho} j_{\rho}^{\prime}}$ and $\quad Q_{j_{\rho} j_{\rho}^{\prime}}=\left\langle l \frac{1}{2} j_{\rho}\right.$ $\left.\| Y^{(2)}|| l^{\prime} \frac{1}{2} j_{\rho}^{\prime}\right\rangle$ represents the matrix element of the fermionic quadrupole operator in the considered single-particle basis. 
TABLE I. The adopted parameters of the IBM-2 Hamiltonian $\hat{H}_{\mathrm{B}}$ for the even-even-core nuclei ${ }^{124-134} \mathrm{Xe}$ and ${ }^{126-136} \mathrm{Ba}$.

\begin{tabular}{lcccc}
\hline \hline & $\epsilon(\mathrm{MeV})$ & $\kappa(\mathrm{MeV})$ & $\chi_{\nu}$ & $\chi_{\pi}$ \\
\hline${ }^{124} \mathrm{Xe}$ & 0.45 & -0.336 & 0.40 & -0.50 \\
${ }^{126} \mathrm{Xe}$ & 0.52 & -0.323 & 0.25 & -0.50 \\
${ }^{128} \mathrm{Xe}$ & 0.62 & -0.315 & 0.25 & -0.55 \\
${ }^{130} \mathrm{Xe}$ & 0.82 & -0.308 & 0.38 & -0.50 \\
${ }^{132} \mathrm{Xe}$ & 0.90 & -0.250 & 0.20 & -0.55 \\
${ }^{134} \mathrm{Xe}$ & 0.98 & -0.190 & 0.20 & -0.60 \\
${ }^{126} \mathrm{Ba}$ & 0.28 & -0.284 & 0.12 & -0.49 \\
${ }^{128} \mathrm{Ba}$ & 0.41 & -0.286 & 0.12 & -0.53 \\
${ }^{130} \mathrm{Ba}$ & 0.52 & -0.297 & 0.25 & -0.55 \\
${ }^{132} \mathrm{Ba}$ & 0.65 & -0.288 & 0.25 & -0.45 \\
${ }^{134} \mathrm{Ba}$ & 0.84 & -0.278 & 0.40 & -0.45 \\
${ }^{136} \mathrm{Ba}$ & 1.00 & -0.278 & 0.40 & -0.45 \\
\hline \hline
\end{tabular}

The exchange term $\hat{V}_{\rho^{\prime} \rho}$ in Eq. (4) reads

$$
\begin{aligned}
\hat{V}_{\rho^{\prime} \rho}= & -\left(s_{\rho^{\prime}}^{\dagger} \tilde{d}_{\rho^{\prime}}\right)^{(2)}\left\{\sum_{j_{\rho} j_{\rho}^{\prime} j_{\rho}^{\prime \prime}} \sqrt{\frac{10}{N_{\rho}\left(2 j_{\rho}+1\right)}} \beta_{j_{\rho} j_{\rho}^{\prime}} \beta_{j_{\rho}^{\prime \prime} j_{\rho}}\right. \\
& \left.\left.:\left[\left(d_{\rho}^{\dagger} \tilde{a}_{j_{\rho}^{\prime \prime}}\right)^{\left(j_{\rho}\right)}\left(a_{j_{\rho}^{\prime}}^{\dagger} \tilde{s}_{\rho}\right)^{\left(j_{\rho}^{\prime}\right)}\right]^{(2)}:\right\}+ \text { (H.c. }\right),
\end{aligned}
$$

with $\beta_{j_{\rho} j_{\rho}^{\prime}}=\left(u_{j_{\rho}} v_{j_{\rho}^{\prime}}+v_{j_{\rho}} u_{j_{\rho}^{\prime}}\right) Q_{j_{\rho} j_{\rho}^{\prime}}$. In the second line of the above equation, the notation : $(\cdots)$ : indicates normal ordering. The definition of the number operator for the odd fermion in the monopole interaction has already been introduced in Eq. (3).

\section{B. Procedure to build the IBFM Hamiltonian}

To build the IBFM Hamiltonian, we first carry out constrained Hartree-Fock-Bogoliubov (HFB) calculations to obtain the potential energy surface (PES), as a function of the quadrupole deformation parameters $\beta$ and $\gamma$, for a set of even-even $\mathrm{Xe}$ and $\mathrm{Ba}$ nuclei. For each nucleus, the parameters $\epsilon, \kappa, \chi_{\nu}$, and $\chi_{\pi}$ of the boson IBM-2 Hamiltonian are fitted to reproduce the HFB PES when the IBM-2 enery is computed using the boson coherent state [43] (see Refs. [44,45], for details).

Next, the single-particle energies $\epsilon_{j_{v}}\left(\epsilon_{j_{\pi}}\right)$ and occupation probabilities $v_{j_{v}}^{2}\left(v_{j_{\pi}}^{2}\right)$ of the unpaired neutron and/or proton are computed with the help of Gogny-D1M HFB calculations constrained to zero deformation [31]. These parameters are used in the $\hat{H}_{\mathrm{F}}^{v}\left(\hat{H}_{\mathrm{F}}^{\pi}\right)$ and $\hat{H}_{\mathrm{BF}}^{v}\left(\hat{H}_{\mathrm{BF}}^{\pi}\right)$ Hamiltonians, respectively. The optimal values of the strength parameters for the boson-fermion Hamiltonian $\hat{H}_{\mathrm{BF}}^{v}\left(\hat{H}_{\mathrm{BF}}^{\pi}\right)$, i.e., $\Gamma_{\nu}, \Lambda_{\nu}$, and $A_{v}\left(\Gamma_{\pi}, \Lambda_{\pi}\right.$, and $\left.A_{\pi}\right)$, are determined separately for positive- and negative-parity states, so as to reproduce the experimental low-energy levels for each of the considered odd $-N$ Xe and $\mathrm{Ba}$ (odd- $\mathrm{Z} \mathrm{Cs}$ and $\mathrm{La}$ ) isotopes. The values of the IBM-2 parameters and the IBFM strengths obtained for the studied even-even and odd- $A$ nuclei are given in Tables I and II, respectively. The IBFM parameters, shown in Table II, are exactly the same as the ones employed in
TABLE II. Strength parameters of the boson-fermion Hamiltonian $\hat{H}_{\mathrm{BF}}^{\rho}$ (in MeV) employed for the studied odd- $A$ nuclei.

\begin{tabular}{lccc}
\hline \hline & $\Gamma_{\rho}$ & $\Lambda_{\rho}$ & $A_{\rho}$ \\
\hline${ }^{123} \mathrm{Xe}$ & 3.20 & 0.20 & -0.14 \\
${ }^{125} \mathrm{Xe}$ & 3.00 & 0.40 & -0.12 \\
${ }^{127} \mathrm{Xe}$ & 3.00 & 0.60 & -0.28 \\
${ }^{129} \mathrm{Xe}$ & 1.60 & 2.20 & -0.30 \\
${ }^{131} \mathrm{Xe}$ & 1.00 & 2.00 & -0.30 \\
${ }^{133} \mathrm{Xe}$ & 0.30 & 2.00 & -0.30 \\
${ }^{125} \mathrm{Ba}$ & 3.00 & 1.55 & 0.0 \\
${ }^{127} \mathrm{Ba}$ & 3.00 & 0.60 & -0.35 \\
${ }^{129} \mathrm{Ba}$ & 1.60 & 1.50 & -0.80 \\
${ }^{131} \mathrm{Ba}$ & 1.20 & 1.80 & -0.50 \\
${ }^{133} \mathrm{Ba}$ & 1.00 & 1.60 & -0.55 \\
${ }^{135} \mathrm{Ba}$ & 0.30 & 1.60 & -0.50 \\
${ }^{125} \mathrm{Cs}$ & 0.80 & 0.51 & -0.80 \\
${ }^{127} \mathrm{Cs}$ & 0.80 & 0.40 & -0.70 \\
${ }^{129} \mathrm{Cs}$ & 1.00 & 0.40 & -0.80 \\
${ }^{131} \mathrm{Cs}$ & 1.20 & 0.55 & -0.50 \\
${ }^{133} \mathrm{Cs}$ & 1.20 & 0.58 & -0.10 \\
${ }^{135} \mathrm{Cs}$ & 0.80 & 1.00 & -2.7 \\
${ }^{127} \mathrm{La}$ & 1.00 & 1.50 & -2.0 \\
${ }^{129} \mathrm{La}$ & 0.80 & 1.76 & -2.3 \\
${ }^{131} \mathrm{La}$ & 0.80 & 1.92 & -1.1 \\
${ }^{133} \mathrm{La}$ & 1.00 & 2.00 & 0.45 \\
${ }^{135} \mathrm{La}$ & 1.50 & 0.81 & 1.45 \\
${ }^{137} \mathrm{La}$ & 2.00 & & \\
\hline \hline
\end{tabular}

Ref. [39]. The spherical single-particle energies and occupation probabilities for these odd- $A$ nuclei can be found in Ref. [34].

The resulting IBFM Hamiltonian is then diagonalized in the basis $\left.\left.\mid\left[L_{v} \otimes L_{\pi}\right]^{(L)} \otimes j_{\rho}\right]^{(I)}\right\rangle$, where $L_{\rho}$ is the angular momentum of the neutron or proton boson system, $L$ is the total angular momentum of the boson system, and $I$ represents the total angular momentum of the coupled boson-fermion system.

\section{Electromagnetic transition operators}

The electromagnetic transition rates in odd- $A$ nuclei can be computed using the eigenstates of the IBFM Hamiltonian. Here, we consider the electric quadrupole $(E 2)$ and magnetic dipole $(M 1)$ properties. The $E 2$ operator $\hat{T}^{(\mathrm{E} 2)}$ reads $[21,39]$

$$
\begin{aligned}
\hat{T}^{(E 2)}= & e_{\nu}^{\mathrm{B}} \hat{Q}_{\nu}+e_{\pi}^{\mathrm{B}} \hat{Q}_{\pi}-\frac{1}{\sqrt{5}} \sum_{\rho=v, \pi} \sum_{j_{\rho} j_{\rho}^{\prime}} \\
& \times\left(u_{j_{\rho}} u_{j_{\rho}^{\prime}}-v_{j_{\rho}} v_{j_{\rho}^{\prime}}\right)\left\langle j_{\rho}^{\prime} \|\left|e_{\rho}^{\mathrm{F}} r^{2} Y^{(2)}\right| \mid j_{\rho}\right\rangle\left(a_{j_{\rho}}^{\dagger} \tilde{a}_{j_{\rho}^{\prime}}\right)^{(2)},
\end{aligned}
$$

where $e_{\rho}^{\mathrm{B}}$ and $e_{\rho}^{\mathrm{F}}$ are the effective charges for the boson and fermion systems, respectively. We have used the fixed values $e_{v}^{\mathrm{B}}=e_{\pi}^{\mathrm{B}}=0.108 \mathrm{eb}$, and $e_{v}^{\mathrm{F}}=0.5 \mathrm{eb}$ and $e_{\pi}^{\mathrm{F}}=1.5 \mathrm{eb}$. These values have already been employed in previous IBFM 
calculations [21] for the same mass region. The $M 1$ transition operator $\hat{T}^{(\mathrm{M} 1)}$ reads [21,39]

$$
\begin{aligned}
\hat{T}^{(M 1)}= & \sqrt{\frac{3}{4 \pi}}\left\{g_{v}^{\mathrm{B}} \hat{L}_{v}^{\mathrm{B}}+g_{\pi}^{\mathrm{B}} \hat{L}_{\pi}^{\mathrm{F}}-\frac{1}{\sqrt{3}} \sum_{\rho=v, \pi} \sum_{j_{\rho} j_{\rho}^{\prime}}\right. \\
& \left.\times\left(u_{j_{\rho}} u_{j_{\rho}^{\prime}}+v_{j_{\rho}} v_{j_{\rho}^{\prime}}\right)\left\langle j_{\rho}^{\prime}|| g_{l}^{\rho} \mathbf{l}+g_{s}^{\rho} \mathbf{s}|| j_{\rho}\right\rangle\left(a_{j_{\rho}}^{\dagger} \tilde{a}_{j_{\rho}^{\prime}}\right)^{(1)}\right\} .
\end{aligned}
$$

In this expression, $g_{v}^{\mathrm{B}}$ and $g_{\pi}^{\mathrm{B}}$ are the $g$ factors for the neutron and proton bosons, respectively. The empirical values $g_{v}^{\mathrm{B}}=$ $0 \mu_{N}$ and $g_{\pi}^{\mathrm{B}}=0.8 \mu_{N}$, taken from Ref. [21], are used for all the studied odd- $A$ nuclei. For the neutron (proton) $g$ factors, the usual Schmidt values $g_{l}^{v}=0 \mu_{N}$ and $g_{s}^{v}=-3.82 \mu_{N}$ $\left(g_{l}^{\pi}=1.0 \mu_{N}\right.$ and $\left.g_{s}^{\pi}=5.58 \mu_{N}\right)$ are used. The $g_{s}$ values, for both protons and neutrons, have been quenched by $30 \%$.

\section{Gamow-Teller and Fermi transition operators}

To obtain the $\beta$-decay $\log f t$ values, the Gamow-Teller (GT) and Fermi (F) matrix elements using the wave functions corresponding to the initial state (with spin $\left|I_{\mathrm{i}}\right\rangle$ ) for the parent nucleus and the final state (with spin $\left|I_{\mathrm{f}}\right\rangle$ ) for the daughter nucleus are needed. Those wave functions are obtained with two independent IBFM calculations. The GT and F operators have to be defined in the boson-fermion space of the IBFM. To this end, we introduce the one-fermion transfer operators [17]:

$$
A_{m}^{(j) \dagger}=\zeta_{j} a_{j m}^{\dagger}+\sum_{j^{\prime}} \zeta_{j j^{\prime}} s_{\rho}^{\dagger}\left(\tilde{d}_{\rho} a_{j^{\prime}}^{\dagger}\right)_{m}^{(j)}\left(\Delta n_{j}=1, \Delta N_{\rho}=0\right)
$$

and

$$
B_{m}^{(j) \dagger}=\theta_{j} s_{\rho}^{\dagger} \tilde{a}_{j m}+\sum_{j^{\prime}} \theta_{j j^{\prime}}\left(d_{\rho}^{\dagger} \tilde{a}_{j^{\prime}}\right)_{m}^{(j)}\left(\Delta n_{j}=-1, \Delta N_{\rho}=1\right) .
$$

Both operators increase the number of valence neutrons (protons) $n_{j}+2 N_{\rho}$ by one. Note, that the index of $j_{\rho}$ is omitted for the sake of simplicity. The conjugate operators read

$$
\begin{aligned}
\tilde{A}_{m}^{(j)} & =(-1)^{j-m}\left\{A_{-m}^{(j) \dagger}\right\}^{\dagger} \\
& =\zeta_{j}^{*} \tilde{a}_{j m}+\sum_{j^{\prime}} \zeta_{j j^{\prime}}^{*} s_{\rho}\left(d_{\rho}^{\dagger} \tilde{a}_{j^{\prime}}\right)_{m}^{(j)}\left(\Delta n_{j}=-1, \Delta N_{\rho}=0\right)
\end{aligned}
$$

and

$$
\begin{aligned}
\tilde{B}_{m}^{(j)} & =(-1)^{j-m}\left\{B_{-m}^{(j) \dagger}\right\}^{\dagger} \\
& =-\theta_{j}^{*} s_{\rho} a_{j m}^{\dagger}-\sum_{j^{\prime}} \theta_{j j^{\prime}}^{*}\left(\tilde{d}_{\rho} a_{j^{\prime}}^{\dagger}\right)_{m}^{(j)}\left(\Delta n_{j}=1, \Delta N_{\rho}=-1\right) .
\end{aligned}
$$

These operators decrease the number of valence neutrons (protons) $n_{j}+2 N_{\rho}$ by one.

The coefficients $\zeta_{j}, \zeta_{j j^{\prime}}, \theta_{j}$, and $\theta_{j j^{\prime}}$ in Eqs. (8)-(11) are given [19] by

$$
\begin{aligned}
\zeta_{j} & =u_{j} \frac{1}{K_{j}^{\prime}}, \\
\zeta_{j j^{\prime}} & =-v_{j} \beta_{j^{\prime} j} \sqrt{\frac{10}{N_{\rho}(2 j+1)}} \frac{1}{K K_{j}^{\prime}}, \\
\theta_{j} & =\frac{v_{j}}{\sqrt{N_{\rho}}} \frac{1}{K_{j}^{\prime \prime}}, \\
\theta_{j j^{\prime}} & =u_{j} \beta_{j^{\prime} j} \sqrt{\frac{10}{2 j+1}} \frac{1}{K K_{j}^{\prime \prime}} .
\end{aligned}
$$

The parameters $K, K_{j}^{\prime}$, and $K_{j}^{\prime \prime} \operatorname{read}[17,19]$

$$
\begin{aligned}
K & =\left(\sum_{j j^{\prime}} \beta_{j j^{\prime}}^{2}\right)^{1 / 2}, \\
K_{j}^{\prime} & =\left[1+2\left(\frac{v_{j}}{u_{j}}\right)^{2} \frac{\left\langle\left(\hat{n}_{s_{\rho}}+1\right) \hat{n}_{d_{\rho}}\right\rangle_{0_{1}^{+}}}{N_{\rho}(2 j+1)} \frac{\sum_{j^{\prime}} \beta_{j^{\prime} j}^{2}}{K^{2}}\right]^{1 / 2}, \\
K_{j}^{\prime \prime} & =\left[\frac{\left\langle\hat{n}_{s_{\rho}}\right\rangle_{0_{1}^{+}}}{N_{\rho}}+2\left(\frac{u_{j}}{v_{j}}\right)^{2} \frac{\left\langle\hat{n}_{d_{\rho}}\right\rangle_{0_{1}^{+}}}{2 j+1} \frac{\sum_{j^{\prime}} \beta_{j^{\prime} j}^{2}}{K^{2}}\right]^{1 / 2} .
\end{aligned}
$$

Note that $\hat{n}_{s_{\rho}}$ is the number operator for the $s_{\rho}$ boson and that $\langle\cdots\rangle_{0_{1}^{+}}$represents the expectation value of a given operator in the $0_{1}^{+}$ground state of the considered even-even nucleus. For a more detailed account, the reader is referred to Refs. [17,19].

With the previously defined building blocks, the IBFM images of the Fermi $\left(\sum_{k} t_{k}^{ \pm}\right)$, and Gamow-Teller $\left(\sum_{k} t_{k}^{ \pm} \sigma_{k}\right)$ transition operators take the form

$$
\begin{aligned}
\hat{\mathcal{O}}^{\mathrm{F}} & =-\sum_{j} \sqrt{2 j+1}\left(P_{v}^{(j)} P_{\pi}^{(j)}\right)^{(0)}, \\
\hat{\mathcal{O}}^{\mathrm{GT}} & =\sum_{j^{\prime} j} \eta_{j^{\prime} j}\left(P_{v}^{\left(j^{\prime}\right)} P_{\pi}^{(j)}\right)^{(1)},
\end{aligned}
$$

where

$$
\begin{aligned}
\eta_{j^{\prime} j} & =-\frac{1}{\sqrt{3}}\left\langle\ell^{\prime} \frac{1}{2} ; j^{\prime}\|\sigma\| \ell \frac{1}{2} ; j\right\rangle \\
& =-\delta_{\ell^{\prime} \ell} \sqrt{2\left(2 j^{\prime}+1\right)(2 j+1)} W\left(\ell j^{\prime} \frac{1}{2} 1 ; \frac{1}{2} j\right),
\end{aligned}
$$

with $W$ being a Racah coefficient. In the case of $\beta^{+}$decay, $P_{v}^{\left(j^{\prime}\right)}=\tilde{B}_{v}^{\left(j^{\prime}\right)}$ and $P_{\pi}^{(j)}=\tilde{A}_{\pi}^{(j)}$, while for $\beta^{-}$decay $P_{v}^{\left(j^{\prime}\right)}=B_{v}^{\left(j^{\prime}\right) \dagger}$ and $P_{\pi}^{(j)}=A_{\pi}^{(j) \dagger}$. Then, the reduced Fermi $B\left(\mathrm{~F} ; I_{\mathrm{i}} \rightarrow I_{\mathrm{f}}\right)$ and 

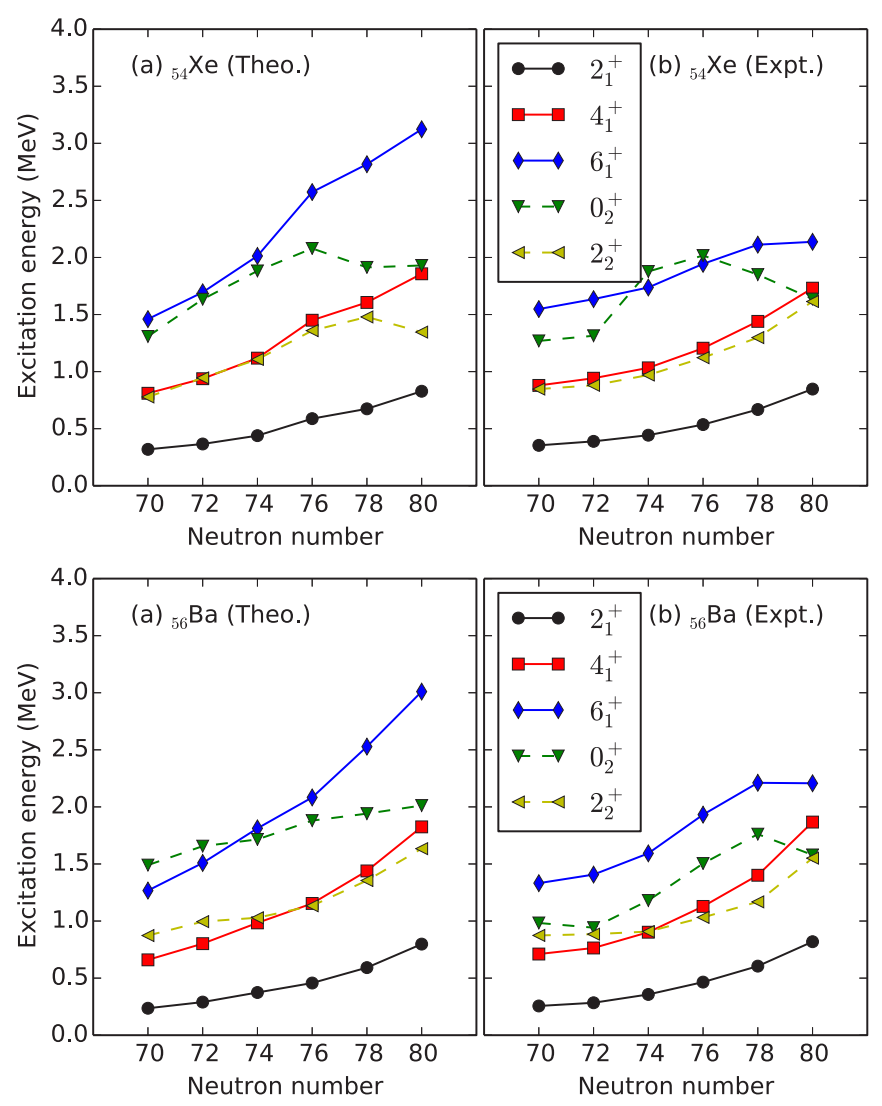

FIG. 1. The low-lying excitation spectra, obtained for the eveneven nuclei ${ }^{124-134} \mathrm{Xe}$ and ${ }^{126-136} \mathrm{Ba}$ with the mapped IBM-2 Hamiltonian, are compared with the corresponding experimental data [47] taken from the ENSDF database.

GT $B\left(\mathrm{GT} ; I_{\mathrm{i}} \rightarrow I_{\mathrm{f}}\right)$ transition rates read

$$
\begin{aligned}
B\left(\mathrm{~F} ; I_{\mathrm{i}}\right. & \left.\rightarrow I_{\mathrm{f}}\right)=\frac{1}{2 I_{\mathrm{i}}+1}\left|\left\langle I_{\mathrm{f}} \| \hat{\mathcal{O}}^{\mathrm{F}}|| I_{\mathrm{i}}\right\rangle\right|^{2}, \\
B\left(\mathrm{GT} ; I_{\mathrm{i}} \rightarrow I_{\mathrm{f}}\right) & =\frac{1}{2 I_{\mathrm{i}}+1}\left|\left\langle I_{\mathrm{f}} \| \hat{\mathcal{O}}^{\mathrm{GT}}|| I_{\mathrm{i}}\right\rangle\right|^{2} .
\end{aligned}
$$

The $\log f t$ for the $\beta$ decay $I_{\mathrm{i}} \rightarrow I_{\mathrm{f}}$, can be computed as

$$
\log f t=\log _{10}\left\{\frac{6163}{B\left(\mathrm{~F} ; I_{\mathrm{i}} \rightarrow I_{\mathrm{f}}\right)+g_{A}^{2} B\left(\mathrm{GT} ; I_{\mathrm{i}} \rightarrow I_{\mathrm{f}}\right)}\right\} .
$$

Here, $g_{A}$ is the ratio of the axial vector to vector coupling constants, $g_{A}=G_{A} / G_{V}$. We have employed the free nucleon value $g_{A}=1.2701(25)$ [46] for all the studied nuclei without quenching.

\section{EVEN-EVEN NUCLEI}

The low-lying excitation spectra, obtained for $\mathrm{Xe}$ and $\mathrm{Ba}$ nuclei with the mapped IBM-2 Hamiltonian, are compared with the corresponding experimental data [47] in Fig. 1. As can be seen from the figure, the predicted spectra reproduce well the experimental ones for both the $\mathrm{Xe}$ and $\mathrm{Ba}$ isotopic chain. Note that the IBM-2 description of the low-lying energy levels in $\mathrm{Xe}$ and $\mathrm{Ba}$ nuclei obtained in this work is more accurate than in Ref. [34], where no distinction was made between neutron and proton degrees of freedom within the IBM-1 model. From $N=70$ to $N \approx 76$, both the predicted and empirical energy levels exhibit features of $\gamma$-soft nuclei (such as the the $R_{4 / 2}$ between the excitation energies of the $4_{1}^{+}$and $2_{1}^{+}$states close to 2.5 , a low-lying $2_{2}^{+}$level close in energy to the $4_{1}^{+}$level and a $0_{2}^{+}$level close in energy to the $6_{1}^{+}$one). The transition from $\gamma$-soft to vibrational spectra is characterized by the behavior of the $0_{2}^{+}$energy level. It starts to decouple from the $6_{1}^{+}$level from $N=76$ to $N=78$ (in Xe) or from $N=78$ to $N=80$ (in Ba). Triplets are then formed with the $4_{1}^{+}$and $2_{2}^{+}$levels, which is a typical feature of a multiphonon spectrum.

The behavior of the excitation energies in even-even Xe and $\mathrm{Ba}$ nuclei, as functions of the neutron number $N$, is consistent with the gradual changes observed in the topology of the underlying PESs, presented in Fig. 5 of Ref. [34]. Those PESs exhibit a prolate or $\gamma$-soft minimum for the nuclei with $N \leqslant 76$ (in $\mathrm{Xe}$ ) and 78 (in $\mathrm{Ba}$ ), as well as transitions to nearly spherical shapes for $N \geqslant 78$ (in $\mathrm{Xe}$ ) and $N \geqslant 80$ (in $\mathrm{Ba}$ ). For a more detailed account, the reader is referred to Ref. [34].

\section{ODD-A NUCLEI}

The positive-parity low-energy excitation spectra, obtained within the IBFM, for odd- $N$ Xe and Ba as well as odd- $Z$ Cs and La nuclei are compared with the experimental spectra in Figs. 2, 3, 4, and 5, respectively. As can be seen from the figures, the overall description of the empirical energy levels in each odd- $A$ system is very reasonable, in spite of having only used three fitted strength parameters to reproduce them. The evolution of some low-lying states can be associated with a shape transition. For instance, in the Xe isotopic chain (Fig. 2), the ground-state spin switches from $I=1 / 2^{+}$at $N=75$ to $3 / 2^{+}$at $N=77$. This correlates well with the shape transitions observed in the neighboring even-even core nuclei (see Fig. 1). Perhaps, the most notable discrepancy between the calculated and experimental spectra is observed in the case of the $1 / 2_{1}^{+}$state in $\mathrm{La}$ isotopes. The predicted energy levels are too low for $N \leqslant 74$, as compared with their experimental counterparts. However, in most of the La isotopes, the experimental $1 / 2_{1}^{+}$energy level has not been firmly established.

The $B(E 2)$ and $B(M 1)$ transition rates as well as the electric quadrupole $Q(I)$ and magnetic dipole $\mu(I)$ moments corresponding to the lowest positive-parity states in all the considered odd- $A \mathrm{Xe}, \mathrm{Ba}, \mathrm{Cs}$, and La nuclei are compared in Fig. 6 with the available experimental data [47]. The electromagnetic transitions between the lowest-lying states tend to be stronger as the number of valence neutrons (holes) increases toward the middle of the major shell $N \approx 66$. Considerable differences between the computed and experimental $B(E 2)$ and $B(M 1)$ values are observed for the lightest $\mathrm{Xe}$ isotopes. In order to understand these deviations, it is useful to decompose the IBFM wave functions for the relevant states into the single-particle configurations involved. For instance, the $1 / 2_{1}^{+}, 3 / 2_{1}^{+}$, and $5 / 2_{1}^{+}$states for ${ }^{123} \mathrm{Xe}$, where particularly large discrepancies are observed between the calculated 


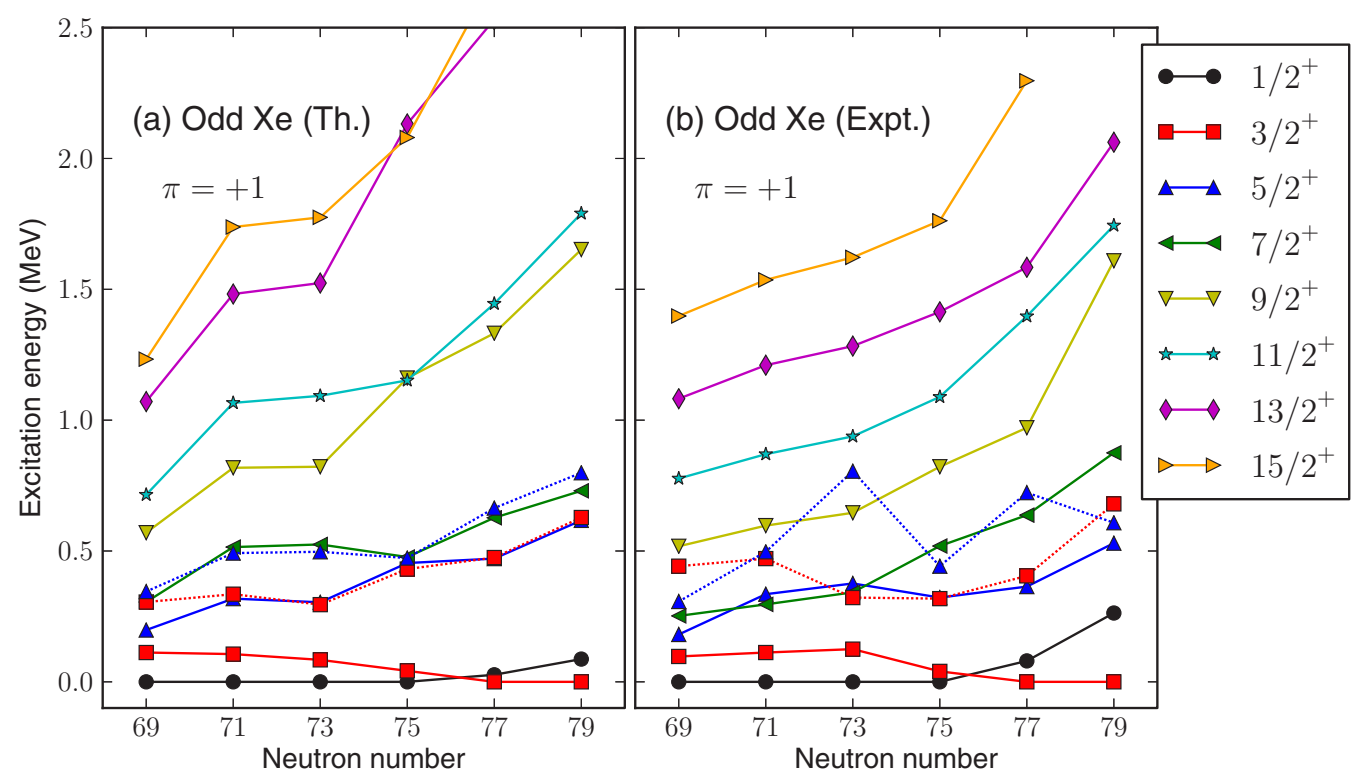

FIG. 2. The calculated positive-parity low-energy excitation spectra obtained for the odd- $A$ isotopes ${ }^{123-133}$ Xe are compared with the experimental data [47] taken from the ENSDF database. The non-yrast $3 / 2_{2}^{+}$and $5 / 2_{2}^{+}$energy levels are connected by broken lines.

and experimental $B(E 2)$ and $B(M 1)$ rates [see Figs. 6(a1) and $6(\mathrm{~b} 1)]$, can be expressed schematically in the following way:

$$
\begin{aligned}
& \left.\left|\frac{1^{+}}{{ }^{+}}\right\rangle=\left.\left[0.68\left|v s_{1 / 2}\right\rangle+0.28\left|v d_{5 / 2}\right\rangle+\cdots\right] \otimes\right|^{124} \mathrm{Xe}\right\rangle, \\
& \left.\left|\frac{3^{+}}{{ }^{+}}\right\rangle=\left.\left[0.67\left|v d_{3 / 2}\right\rangle+0.16\left|v g_{7 / 2}\right\rangle+\cdots\right] \otimes\right|^{124} \mathrm{Xe}\right\rangle, \\
& \left|\frac{5}{2}_{1}^{+}\right\rangle=\left[0.45\left|v s_{1 / 2}\right\rangle+0.49\left|v d_{5 / 2}\right\rangle+\cdots\right] \otimes\left|{ }^{124} \mathrm{Xe}\right\rangle,
\end{aligned}
$$

where the components with amplitudes smaller than 0.1 have been omitted. The $1 / 2_{1}^{+}$and $5 / 2_{1}^{+}$states appear to be similar in structure, i.e., they are mainly made of the $3 s_{1 / 2}$ and $2 d_{5 / 2}$ single-neutron configurations. The large overlap between the states leads to the strong $B(E 2)$ transition that follows the $\Delta I=2$ sequence of the weak coupling limit. However, the $1 / 2_{1}^{+}$and $3 / 2_{1}^{+}$states have different structures leading to the small $B(E 2)$ and $B(M 1)$ transition rates computed between these states. As for the electric quadrupole and magnetic dipole moments, shown in panels Figs. 6(c1)-6(c4) and $6(\mathrm{~d} 1)-6(\mathrm{~d} 4)$, the calculations reproduce reasonably well the experimental data, at least the correct sign, for most of the considered nuclei.


FIG. 3. The same as in Fig. 2, but for the odd- $A$ isotopes ${ }^{125-135} \mathrm{Ba}$. 


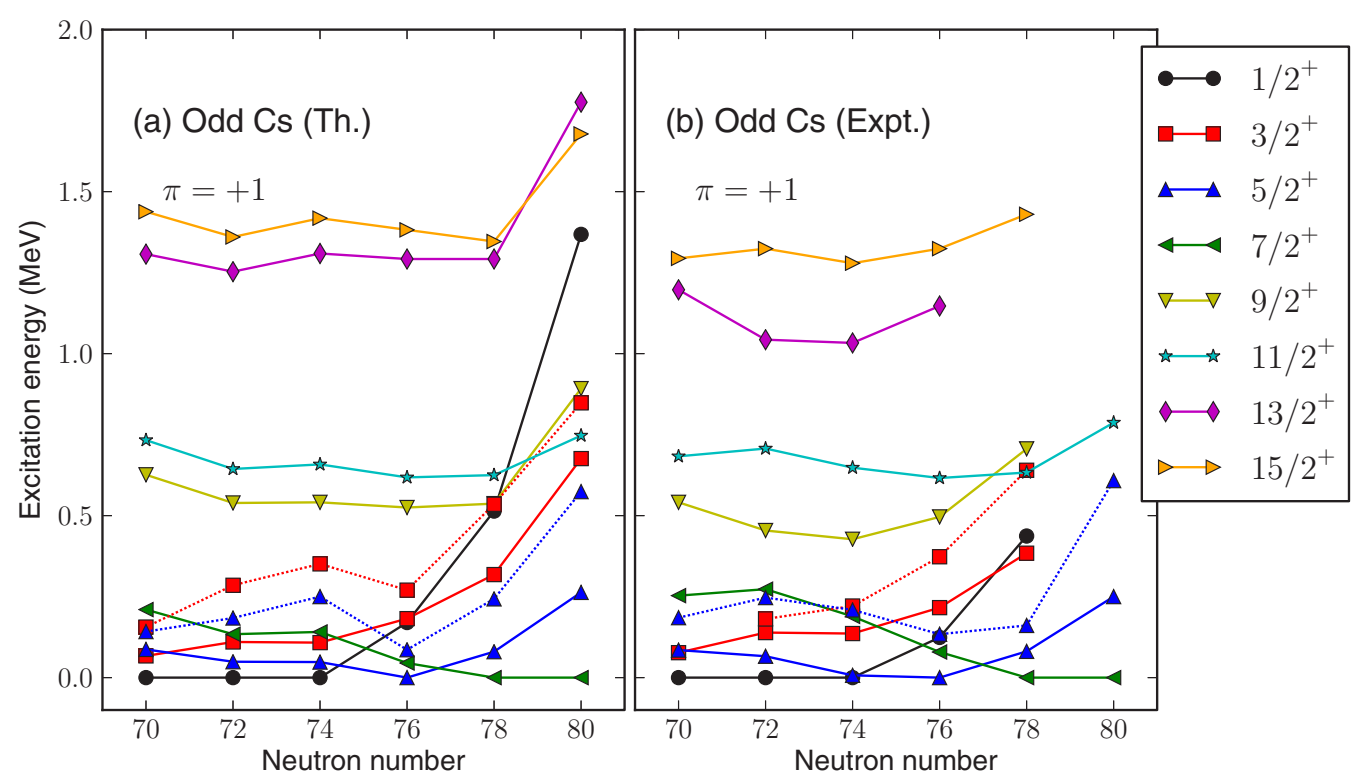

FIG. 4. The same as in Fig. 2, but for the odd- $A$ isotopes ${ }^{125-135}$ Cs.

\section{V. $\beta$ DECAY}

\section{A. Overall results}

Having obtained a reasonable global description of the energies and transition properties in the even-even and odd- $A$ nuclei, we now turn to the discussion of the $\beta$ decay. We have computed the $\log f t$ values of the $\beta^{+}$and $\beta^{-}$decays of those odd- $A$ nuclei, for which the experimental data are available. We will restrict our discussion to those $\beta$ decays where only GT and $\mathrm{F}$ transitions are involved, i.e., with $\Delta I=0, \pm 1$ and no parity change. Note that most of the observed $\beta$ decays in this region of the nuclear chart are of this type. In Fig. 7(a), we have plotted the $f t$ values corresponding to the $\beta^{+}$decay of the odd- $A$ nuclei ${ }^{125-131} \mathrm{Cs},{ }^{127-133} \mathrm{Ba}$, and ${ }^{129-135} \mathrm{La}$ as well as the one corresponding to the $\beta^{-}$decay of ${ }^{133} \mathrm{Xe}$. The transitions shown in the figure are those from the ground state of the parent nucleus to the lowest-energy state of daughter nucleus, for which experimental data are available.

The theoretical to experimental ratio for the $f t$ values, $f t^{\text {th }} / f t^{\mathrm{ex}}$, is depicted in Fig. 7(b). The theoretical $f t$ values $f t^{\text {th }}$ increase as the neutron shell closure $N=82$ is approached. In most of the cases, the computed $\beta$-decay $f t$ values underestimate the experimental ones, with the largest deviation for the $\mathrm{Cs} \longrightarrow$ Xe decays. In our calculation, the boson-core Hamiltonian, the single-particle energies, and the occupation probabilities for the odd nucleon have been


FIG. 5. The same as in Fig. 2, but for the odd- $A$ isotopes ${ }^{127-137}$ La. 



FIG. 6. The $B(E 2)$ and $B(M 1)$ transition rates (in Weisskopf units) as well as the electric quadrupole $Q(I)$ (in $e$ b) and magnetic dipole $\mu(I)$ (in nuclear magneton $\mu_{N}$ ) moments corresponding to the lowest positive-parity states in the studied Xe [panels (a1)-(d1)], Ba [panels (a2)-(d2)], Cs [panels (a3)-(d3)], and La [panels (a4)-(d4)] nuclei are compared with the experimental data [47]. Those experimental B(E2) and $B(M 1)$ values without error bars are the lower limits. For the $B(E 2)$ rates, the theoretical and experimental values for the odd- $N$ Xe and Ba (odd- $Z$ Cs and La) nuclei are defined in panels (a1) and (a2) [panels (a3) and (a4)], respectively. The same rule applies to those other properties shown in the figure.

obtained via (constrained) fully microscopic Gogny-D1M HFB calculations. In addition, no phenomenological parameter has been introduced to compute the GT and F matrix elements. In view of these, the description of the observed $\log f t$ values by the present IBFM calculation is reasonably good. Let us also stress that no normalization factor has been introduced for the GT or the F matrix elements, as is usually done, for example, in shell-model calculations. 




FIG. 7. The $f t$ values corresponding to the $\beta^{+}$decay of the odd- $A$ nuclei ${ }^{125-131} \mathrm{Cs}$, ${ }^{127-133} \mathrm{Ba}$, and ${ }^{129-135} \mathrm{La}$ as well as the one corresponding to the $\beta^{-}$decay of ${ }^{133} \mathrm{Xe}$ are plotted in panel (a). The theoretical (experimental) values are represented by open (filled) symbols connected by solid (broken) lines. The ratios $f t^{\text {th }} / f t^{\mathrm{ex}}$ of the predicted to the experimental $f t$ values are depicted in panel (b).

\section{B. $\boldsymbol{\beta}$ decays of odd- $\boldsymbol{A}$ Cs and Xe nuclei}

Let us now turn our attention to the detailed comparison between the computed and experimental $\log f t$ values for the $\beta^{ \pm}$decays of the odd- $A$ Cs and Xe (Table III). In the case of the odd- $A$ Ba isotopes, the $\beta^{+}$decays or electron captures (EC) are presented in Table IV. The corresponding results for the odd- $A$ La nuclei can be found in Tables V and VI. In each table, we have included the computed $\log f t$ values for transitions from an initial state to a selected set of final states (up to five states with the lowest energies for a given spin). There are uncertainties in the experimental determination of the spin of many nearly degenerate excited states that make difficult to establish a correspondence with the states obtained in the calculation. In those cases, we use a footnote in the experimental $\log f t$ value to indicate the possible spins and parities.

For the $\beta$ decays of the odd- $A$ Cs and Xe nuclei, the predicted $\log f t$ values in Table III are systematically smaller
TABLE III. The calculated and experimental $\log f t$ values for the $\beta^{+} / \mathrm{EC}$ (electron-capture) decay of the odd- $A$ Cs into Xe nuclei. Results are also included for the $\beta^{-}$decay of ${ }^{133} \mathrm{Xe}$ into ${ }^{133} \mathrm{Cs}$. Experimental data are taken from Ref. [47].

\begin{tabular}{|c|c|c|c|}
\hline \multirow[b]{2}{*}{ Decay } & \multirow[b]{2}{*}{$I_{\mathrm{i}} \rightarrow I_{\mathrm{f}}$} & \multicolumn{2}{|c|}{$\log f t$} \\
\hline & & Theory & Experiment \\
\hline \multirow[t]{8}{*}{${ }^{125} \mathrm{Cs} \rightarrow{ }^{125} \mathrm{Xe}$} & $1 / 2_{1}^{+} \rightarrow 1 / 2_{1}^{+}$ & 3.725 & $\approx 5.60$ \\
\hline & $1 / 2_{1}^{+} \rightarrow 1 / 2_{2}^{+}$ & 4.608 & $\approx 5.53^{\mathrm{a}}$ \\
\hline & $1 / 2_{1}^{+} \rightarrow 1 / 2_{3}^{+}$ & 6.393 & $\approx 6.96^{\mathrm{b}}$ \\
\hline & $1 / 2_{1}^{+} \rightarrow 3 / 2_{1}^{+}$ & 5.871 & $\approx 6.76$ \\
\hline & $1 / 2_{1}^{+} \rightarrow 3 / 2_{2}^{+}$ & 4.820 & $\approx 5.53^{\mathrm{a}}$ \\
\hline & $1 / 2_{1}^{+} \rightarrow 3 / 2_{3}^{+}$ & 4.771 & $\approx 6.38$ \\
\hline & $1 / 2_{1}^{+} \rightarrow 3 / 2_{4}^{+}$ & 5.278 & $\approx 6.08$ \\
\hline & $1 / 2_{1}^{+} \rightarrow 3 / 2_{5}^{+}$ & 5.045 & $\approx 6.96^{\mathrm{b}}$ \\
\hline \multirow[t]{9}{*}{${ }^{127} \mathrm{Cs} \rightarrow{ }^{127} \mathrm{Xe}$} & $1 / 2_{1}^{+} \rightarrow 1 / 2_{1}^{+}$ & 3.711 & $6.53(6)$ \\
\hline & $1 / 2_{1}^{+} \rightarrow 1 / 2_{2}^{+}$ & 4.628 & $5.558(11)$ \\
\hline & $1 / 2_{1}^{+} \rightarrow 1 / 2_{3}^{+}$ & 5.151 & $7.305(18)^{\mathrm{c}}$ \\
\hline & $1 / 2_{1}^{+} \rightarrow 1 / 2_{4}^{+}$ & 5.984 & $8.02(3)^{\mathrm{d}}$ \\
\hline & $1 / 2_{1}^{+} \rightarrow 3 / 2_{1}^{+}$ & 8.405 & $6.791(24)$ \\
\hline & $1 / 2_{1}^{+} \rightarrow 3 / 2_{2}^{+}$ & 4.426 & $7.574(20)$ \\
\hline & $1 / 2_{1}^{+} \rightarrow 3 / 2_{3}^{+}$ & 5.127 & $8.83(10)$ \\
\hline & $1 / 2_{1}^{+} \rightarrow 3 / 2_{4}^{+}$ & 5.563 & $6.306(12)$ \\
\hline & $1 / 2_{1}^{+} \rightarrow 3 / 2_{5}^{+}$ & 5.417 & $6.988(18)$ \\
\hline \multirow[t]{8}{*}{${ }^{129} \mathrm{Cs} \rightarrow{ }^{129} \mathrm{Xe}$} & $1 / 2_{1}^{+} \rightarrow 1 / 2_{1}^{+}$ & 4.318 & $6.27(6)$ \\
\hline & $1 / 2_{1}^{+} \rightarrow 1 / 2_{2}^{+}$ & 4.007 & $5.68(3)$ \\
\hline & $1 / 2_{1}^{+} \rightarrow 1 / 2_{3}^{+}$ & 4.201 & $6.80(4)^{\mathrm{e}}$ \\
\hline & $1 / 2_{1}^{+} \rightarrow 3 / 2_{1}^{+}$ & 6.728 & $7.3(2)$ \\
\hline & $1 / 2_{1}^{+} \rightarrow 3 / 2_{2}^{+}$ & 5.509 & $7.14(3)$ \\
\hline & $1 / 2_{1}^{+} \rightarrow 3 / 2_{3}^{+}$ & 4.694 & $6.50(3)$ \\
\hline & $1 / 2_{1}^{+} \rightarrow 3 / 2_{4}^{+}$ & 5.558 & $7.68(4)$ \\
\hline & $1 / 2_{1}^{+} \rightarrow 3 / 2_{5}^{+}$ & 6.403 & $6.80(4)^{\mathrm{e}}$ \\
\hline${ }^{131} \mathrm{Cs} \rightarrow{ }^{131} \mathrm{Xe}$ & $5 / 2_{1}^{+} \rightarrow 3 / 2_{1}^{+}$ & 6.116 & $5.548(14)$ \\
\hline \multirow[t]{3}{*}{${ }^{133} \mathrm{Xe} \rightarrow{ }^{133} \mathrm{Cs}$} & $3 / 2_{1}^{+} \rightarrow 3 / 2_{1}^{+}$ & 5.224 & $6.86(9)$ \\
\hline & $3 / 2_{1}^{+} \rightarrow 5 / 2_{1}^{+}$ & 5.656 & $5.619(12)$ \\
\hline & $3 / 2_{1}^{+} \rightarrow 5 / 2_{2}^{+}$ & 8.199 & $7.10(19)$ \\
\hline
\end{tabular}

${ }^{\mathrm{a}} 1 / 2^{(+)}, 3 / 2^{(+)}$level at $525 \mathrm{keV}$ in ${ }^{125} \mathrm{Xe}$.

${ }^{\mathrm{b}} 1 / 2,3 / 2$ level at $1312 \mathrm{keV}$ in ${ }^{125} \mathrm{Xe}$.

${ }^{\mathrm{c}} 1 / 2^{+}, 3 / 2^{+}$level at $1197 \mathrm{keV}$ in ${ }^{127} \mathrm{Xe}$

${ }^{\mathrm{d}} 1 / 2,3 / 2,5 / 2^{+}$level at $1558 \mathrm{keV}$ in ${ }^{127} \mathrm{Xe}$

${ }^{\mathrm{e}} 1 / 2^{+}$or $3 / 2^{+}$level at $946 \mathrm{keV}$ in ${ }^{129} \mathrm{Xe}$

than the experimental ones. The discrepancy could be explained by analyzing the dominant contributions to the GT and $\mathrm{F}$ transition matrix elements. As an example, let us consider the decay of the $1 / 2_{1}^{+}$ground state of ${ }^{125} \mathrm{Cs}$ to the $1 / 2_{1}^{+}$ ground state of ${ }^{125} \mathrm{Xe}$ for which the experimental $f t$ value is underestimated by about a factor of $10^{2}$ [see Eq. (22)].

The reduced GT transition matrix element for this decay is $\left\langle 1 / 2_{1}^{+} \| \hat{\mathcal{O}}^{\mathrm{GT}}|| 1 / 2_{1}^{+}\right\rangle=-1.194$, and the largest contributions come from terms proportional to $s_{v}\left[a_{v s_{1 / 2}}^{\dagger} \tilde{a}_{\pi s_{1 / 2}}\right]^{(1)}$ and $\left[\left(a_{v s_{1 / 2}}^{\dagger} \tilde{d}_{v}\right)^{(j)} \tilde{a}_{\pi d_{5 / 2}}\right]^{(1)}$ (with $j$ being the intermediate angular momentum). The coefficients for these terms are 
TABLE IV. The same as in Table III, but for the $\beta^{+} / \mathrm{EC}$ decays of the odd- $A$ Ba into $C$ s nuclei.

\begin{tabular}{|c|c|c|c|}
\hline \multirow[b]{2}{*}{ Decay } & \multirow[b]{2}{*}{$I_{\mathrm{i}} \rightarrow I_{\mathrm{f}}$} & \multicolumn{2}{|c|}{$\log f t$} \\
\hline & & Theory & Experiment \\
\hline \multirow[t]{7}{*}{${ }^{127} \mathrm{Ba} \rightarrow{ }^{127} \mathrm{Cs}$} & $1 / 2_{1}^{+} \rightarrow 1 / 2_{1}^{+}$ & 6.575 & $5.182(24)$ \\
\hline & $1 / 2_{1}^{+} \rightarrow 1 / 2_{2}^{+}$ & 5.357 & $7.25(15)^{\mathrm{a}}$ \\
\hline & $1 / 2_{1}^{+} \rightarrow 1 / 2_{3}^{+}$ & 5.354 & $7.25(9)^{\mathrm{b}}$ \\
\hline & $1 / 2_{1}^{+} \rightarrow 3 / 2_{1}^{+}$ & 6.736 & $6.81(11)$ \\
\hline & $1 / 2_{1}^{+} \rightarrow 3 / 2_{2}^{+}$ & 5.630 & $5.40(7)$ \\
\hline & $1 / 2_{1}^{+} \rightarrow 3 / 2_{3}^{+}$ & 5.629 & $7.25(15)^{\mathrm{a}}$ \\
\hline & $1 / 2_{1}^{+} \rightarrow 3 / 2_{4}^{+}$ & 6.732 & $7.25(9)^{\mathrm{b}}$ \\
\hline \multirow[t]{7}{*}{${ }^{129} \mathrm{Ba} \rightarrow{ }^{129} \mathrm{Cs}$} & $1 / 2_{1}^{+} \rightarrow 1 / 2_{1}^{+}$ & 6.514 & $5.63(3)$ \\
\hline & $1 / 2_{1}^{+} \rightarrow 1 / 2_{2}^{+}$ & 5.723 & $6.59(5)^{\mathrm{c}}$ \\
\hline & $1 / 2_{1}^{+} \rightarrow 1 / 2_{3}^{+}$ & 5.432 & $6.65(5)^{d}$ \\
\hline & $1 / 2_{1}^{+} \rightarrow 3 / 2_{1}^{+}$ & 8.313 & $6.39(4)$ \\
\hline & $1 / 2_{1}^{+} \rightarrow 3 / 2_{2}^{+}$ & 6.483 & $5.91(3)$ \\
\hline & $1 / 2_{1}^{+} \rightarrow 3 / 2_{3}^{+}$ & 6.043 & $6.59(5)^{c}$ \\
\hline & $1 / 2_{1}^{+} \rightarrow 3 / 2_{4}^{+}$ & 6.992 & $6.65(5)^{d}$ \\
\hline \multirow[t]{8}{*}{${ }^{131} \mathrm{Ba} \rightarrow{ }^{131} \mathrm{Cs}$} & $1 / 2_{1}^{+} \rightarrow 1 / 2_{1}^{+}$ & 6.262 & $8.58(16)$ \\
\hline & $1 / 2_{1}^{+} \rightarrow 1 / 2_{2}^{+}$ & 5.892 & $6.633(8)$ \\
\hline & $1 / 2_{1}^{+} \rightarrow 1 / 2_{3}^{+}$ & 5.305 & $6.66(17)^{\mathrm{e}}$ \\
\hline & $1 / 2_{1}^{+} \rightarrow 3 / 2_{1}^{+}$ & 6.360 & $7.404(11)$ \\
\hline & $1 / 2_{1}^{+} \rightarrow 3 / 2_{2}^{+}$ & 6.729 & $7.305(9)$ \\
\hline & $1 / 2_{1}^{+} \rightarrow 3 / 2_{3}^{+}$ & 6.117 & $8.156(19)$ \\
\hline & $1 / 2_{1}^{+} \rightarrow 3 / 2_{4}^{+}$ & 7.256 & $8.505(11)$ \\
\hline & $1 / 2_{1}^{+} \rightarrow 3 / 2_{5}^{+}$ & 8.046 & $9.78(7)^{\mathrm{f}}$ \\
\hline \multirow[t]{2}{*}{${ }^{133} \mathrm{Ba} \rightarrow{ }^{133} \mathrm{Cs}$} & $1 / 2_{1}^{+} \rightarrow 1 / 2_{1}^{+}$ & 5.530 & $6.627(18)$ \\
\hline & $1 / 2_{1}^{+} \rightarrow 3 / 2_{1}^{+}$ & 6.768 & $8.020(15)$ \\
\hline
\end{tabular}

${ }^{\mathrm{a}} 1 / 2,3 / 2$ level at $568 \mathrm{keV}$ in ${ }^{127} \mathrm{Cs}$.

${ }^{\mathrm{b}} 1 / 2,3 / 2$ level at $713 \mathrm{keV}$ in ${ }^{127} \mathrm{Cs}$.

${ }^{\mathrm{c}}(1 / 2,3 / 2)^{+}$level at $554 \mathrm{keV}$ in ${ }^{129} \mathrm{Cs}$.

${ }^{\mathrm{d}}(1 / 2,3 / 2)^{+}$level at $1165 \mathrm{keV}$ in ${ }^{129} \mathrm{Cs}$.

${ }^{\mathrm{e}} 1 / 2,3 / 2$ level at $1342 \mathrm{keV}$ in ${ }^{131} \mathrm{Cs}$.

${ }^{\mathrm{f}}\left(3 / 2^{+}, 3 / 2^{+}\right)$level at $920 \mathrm{keV}$ in ${ }^{131} \mathrm{Cs}$.

-0.900 and -0.306 , respectively. For the Fermi transition, $\left\langle 1 / 2_{1}^{+} \| \hat{\mathcal{O}}^{\mathrm{F}}|| 1 / 2_{1}^{+}\right\rangle=-0.145$, and the leading terms take the forms $s_{\nu}\left[a_{v s_{1 / 2}}^{\dagger} \tilde{a}_{\pi s_{1 / 2}}\right]^{(0)}$ and $s_{v}\left[a_{v d_{5 / 2}}^{\dagger} \tilde{a}_{\pi d_{5 / 2}}\right]^{(0)}$. Thus, the $3 s_{1 / 2}$ and $2 d_{5 / 2}$ neutron and proton single-particle configurations are dominant components in both the $B\left(\mathrm{GT} ; 1 / 2_{1}^{+} \rightarrow 1 / 2_{1}^{+}\right)$ and $B\left(\mathrm{~F} ; 1 / 2_{1}^{+} \rightarrow 1 / 2_{1}^{+}\right)$values for the ${ }^{125} \mathrm{Cs} \rightarrow{ }^{125} \mathrm{Xe}$ decay. This agrees well, with the fact that the $1 / 2_{1}^{+}$IBFM ground state wave functions for the parent ${ }^{125} \mathrm{Cs}$ and daughter ${ }^{125} \mathrm{Xe}$ systems mainly consist of the $3 s_{1 / 2}$ and $2 d_{5 / 2}$ single-particle configurations. In particular, 31\% (67\%) and $49 \%(18 \%)$ of the $1 / 2_{1}^{+}$wave function in ${ }^{125} \mathrm{Cs}\left({ }^{125} \mathrm{Xe}\right)$ are accounted for by the $3 s_{1 / 2}$ and $2 d_{5 / 2}$ configurations, respectively. The similar wave function contents for the parent and daughter nuclei could partly account for the too large $B(\mathrm{GT})$ and $B(\mathrm{~F})$ values and, therefore, for the smaller $\beta$-decay $\log f t$ values as compared with the experimental data.
TABLE V. The same as in Table III, but for the $\beta^{+} / \mathrm{EC}$ decays of the odd- $A{ }^{129,131}$ La nuclei.

\begin{tabular}{|c|c|c|c|}
\hline \multirow[b]{2}{*}{ Decay } & \multirow[b]{2}{*}{$I_{\mathrm{i}} \rightarrow I_{\mathrm{f}}$} & \multicolumn{2}{|c|}{$\log f t$} \\
\hline & & Theory & Experiment \\
\hline \multirow{15}{*}{${ }^{129} \mathrm{La} \rightarrow{ }^{129} \mathrm{Ba}$} & $3 / 2_{1}^{+} \rightarrow 1 / 2_{1}^{+}$ & 5.286 & $5.89(8)$ \\
\hline & $3 / 2_{1}^{+} \rightarrow 1 / 2_{2}^{+}$ & 6.559 & $5.55(2)$ \\
\hline & $3 / 2_{1}^{+} \rightarrow 1 / 2_{3}^{+}$ & 4.871 & $7.2(1)$ \\
\hline & $3 / 2_{1}^{+} \rightarrow 1 / 2_{4}^{+}$ & 5.477 & $6.83(7)$ \\
\hline & $3 / 2_{1}^{+} \rightarrow 1 / 2_{5}^{+}$ & 9.064 & $6.62(5)$ \\
\hline & $3 / 2_{1}^{+} \rightarrow 3 / 2_{1}^{+}$ & 4.713 & $5.90(5)$ \\
\hline & $3 / 2_{1}^{+} \rightarrow 3 / 2_{2}^{+}$ & 6.058 & $6.23(5)$ \\
\hline & $3 / 2_{1}^{+} \rightarrow 3 / 2_{3}^{+}$ & 6.856 & $5.59(3)$ \\
\hline & $3 / 2_{1}^{+} \rightarrow 3 / 2_{4}^{+}$ & 6.916 & $6.45(4)^{a}$ \\
\hline & $3 / 2_{1}^{+} \rightarrow 3 / 2_{5}^{+}$ & 4.502 & $6.06(3)^{b}$ \\
\hline & $3 / 2_{1}^{+} \rightarrow 5 / 2_{1}^{+}$ & 6.991 & $6.60(5)$ \\
\hline & $3 / 2_{1}^{+} \rightarrow 5 / 2_{2}^{+}$ & 6.149 & $7.3(1)$ \\
\hline & $3 / 2_{1}^{+} \rightarrow 5 / 2_{3}^{+}$ & 7.436 & $6.45(4)^{a}$ \\
\hline & $3 / 2_{1}^{+} \rightarrow 5 / 2_{4}^{+}$ & 7.978 & $6.50(4)$ \\
\hline & $3 / 2_{1}^{+} \rightarrow 5 / 2_{5}^{+}$ & 5.426 & $6.06(3)^{b}$ \\
\hline \multirow[t]{12}{*}{${ }^{131} \mathrm{La} \rightarrow{ }^{131} \mathrm{Ba}$} & $3 / 2_{1}^{+} \rightarrow 1 / 2_{1}^{+}$ & 5.223 & $6.26(9)$ \\
\hline & $3 / 2_{1}^{+} \rightarrow 1 / 2_{2}^{+}$ & 6.710 & $5.82(3)$ \\
\hline & $3 / 2_{1}^{+} \rightarrow 1 / 2_{3}^{+}$ & 5.385 & $6.56(4)^{\mathrm{c}}$ \\
\hline & $3 / 2_{1}^{+} \rightarrow 1 / 2_{4}^{+}$ & 5.114 & $6.43(3)^{\mathrm{d}}$ \\
\hline & $3 / 2_{1}^{+} \rightarrow 3 / 2_{1}^{+}$ & 4.676 & $6.25(5)$ \\
\hline & $3 / 2_{1}^{+} \rightarrow 3 / 2_{2}^{+}$ & 5.857 & $6.34(4)$ \\
\hline & $3 / 2_{1}^{+} \rightarrow 3 / 2_{3}^{+}$ & 7.121 & $5.58(3)$ \\
\hline & $3 / 2_{1}^{+} \rightarrow 3 / 2_{4}^{+}$ & 5.345 & $6.18(3)^{\mathrm{e}}$ \\
\hline & $3 / 2_{1}^{+} \rightarrow 3 / 2_{5}^{+}$ & 6.077 & $6.56(4)^{c}$ \\
\hline & $3 / 2_{1}^{+} \rightarrow 5 / 2_{1}^{+}$ & 7.193 & $6.85(5)$ \\
\hline & $3 / 2_{1}^{+} \rightarrow 5 / 2_{2}^{+}$ & 5.917 & $6.18(3)^{\mathrm{e}}$ \\
\hline & $3 / 2_{1}^{+} \rightarrow 5 / 2_{3}^{+}$ & 5.601 & $6.56(4)^{c}$ \\
\hline
\end{tabular}

${ }^{\mathrm{a}}\left(3 / 2^{+}, 5 / 2^{+}\right)$level at $618 \mathrm{keV}$ in ${ }^{129} \mathrm{Ba}$.

$\mathrm{b}(3 / 2,5 / 2)^{+}$level at $712 \mathrm{keV}$ in ${ }^{129} \mathrm{Ba}$.

${ }^{\mathrm{c}} 1 / 2^{+}, 3 / 2^{+}, 5 / 2^{+}$level at $719 \mathrm{keV}$ in ${ }^{131} \mathrm{Ba}$.

${ }^{\mathrm{d}} 1 / 2^{+}, 3 / 2^{+}, 5 / 2^{+}$level at $879 \mathrm{keV}$ in ${ }^{131} \mathrm{Ba}$.

e $3 / 2^{+}, 5 / 2^{+}$level at $562 \mathrm{keV}$ in ${ }^{131} \mathrm{Ba}$.

\section{C. $\boldsymbol{\beta}$ decays of odd- $\boldsymbol{A}$ Ba nuclei}

As seen from Table IV, the $\log f t$ values predicted for the decays of the odd- $A \mathrm{Ba}$ nuclei are, in general, larger than those for the decays of odd- $A$ Cs (considered in Table III). The overall description of the experimental $\log f t$ values in the cases of the $\mathrm{Ba} \longrightarrow$ Cs decays is, therefore, slightly better than for the $\mathrm{Cs} \longrightarrow$ Xe decays.

We observe that, similar to the cases of the odd- $A$ Cs nuclei, the predicted $\log f t$ values of the odd $A \mathrm{Ba}$ are calculated to be systematically smaller than the empirical values. There are, however, examples of $\beta$ decays of the odd- $A$ Ba systems, for which the theory overestimates the experiment. The largest deviation occurs, for example, in the case of the ${ }^{129} \mathrm{Ba}\left(1 / 2_{1}^{+}\right) \rightarrow{ }^{129} \mathrm{Cs}\left(3 / 2_{1}^{+}\right)$decay. In particular, the computed $\log f t$ is a factor of 1.3 larger than the 
TABLE VI. The same as in Table III, but for the $\beta^{+} / \mathrm{EC}$ decays of the odd- $A{ }^{133,135}$ La nuclei.

\begin{tabular}{lccc}
\hline \hline & & \multicolumn{2}{c}{$\log f t$} \\
\cline { 2 - 3 } Decay & $I_{\mathrm{i}} \rightarrow I_{\mathrm{f}}$ & Theory & Experiment \\
\hline${ }^{133} \mathrm{La} \rightarrow{ }^{133} \mathrm{Ba}$ & $5 / 2_{1}^{+} \rightarrow 3 / 2_{1}^{+}$ & 5.495 & $5.51(4)$ \\
& $5 / 2_{1}^{+} \rightarrow 3 / 2_{2}^{+}$ & 5.420 & $6.97(3)$ \\
$5 / 2_{1}^{+} \rightarrow 3 / 2_{3}^{+}$ & 7.293 & $6.89(3)$ \\
$5 / 2_{1}^{+} \rightarrow 3 / 2_{4}^{+}$ & 7.635 & $7.77(4)$ \\
$5 / 2_{1}^{+} \rightarrow 3 / 2_{5}^{+}$ & 9.694 & $8.48(9)^{\mathrm{a}}$ \\
& $5 / 2_{1}^{+} \rightarrow 5 / 2_{1}^{+}$ & 5.621 & $7.26(5)$ \\
$5 / 2_{1}^{+} \rightarrow 5 / 2_{2}^{+}$ & 5.375 & $7.13(3)$ \\
$5 / 2_{1}^{+} \rightarrow 5 / 2_{3}^{+}$ & 5.192 & $7.80(6)^{\mathrm{b}}$ \\
$5 / 2_{1}^{+} \rightarrow 5 / 2_{4}^{+}$ & 5.490 & $6.71(4)$ \\
$5 / 2_{1}^{+} \rightarrow 5 / 2_{5}^{+}$ & 5.718 & $7.24(4)^{\mathrm{c}}$ \\
$5 / 2_{1}^{+} \rightarrow 7 / 2_{1}^{+}$ & 5.770 & $7.51(4)$ \\
$5 / 2_{1}^{+} \rightarrow 7 / 2_{2}^{+}$ & 5.671 & $8.13(5)$ \\
$5 / 2_{1}^{+} \rightarrow 7 / 2_{3}^{+}$ & 7.105 & $7.24(4)^{\mathrm{c}}$ \\
$5 / 2_{1}^{+} \rightarrow 7 / 2_{4}^{+}$ & 5.498 & $7.36(5)$ \\
$5 / 2_{1}^{+} \rightarrow 3 / 2_{1}^{+}$ & 8.190 & $5.66(1)$ \\
$5 / 2_{1}^{+} \rightarrow 3 / 2_{2}^{+}$ & 5.577 & $7.88(7)$ \\
$5 / 2_{1}^{+} \rightarrow 3 / 2_{3}^{+}$ & 8.599 & $7.75(8)$ \\
$5 / 2_{1}^{+} \rightarrow 3 / 2_{4}^{+}$ & 5.634 & $8.25(9)^{\mathrm{d}}$ \\
$5 / 2_{1}^{+} \rightarrow 5 / 2_{1}^{+}$ & 4.961 & $7.01(7)$ \\
$5 / 2_{1}^{+} \rightarrow 5 / 2_{2}^{+}$ & 5.237 & $8.25(9)^{\mathrm{d}}$ \\
$5 / 2_{1}^{+} \rightarrow 7 / 2_{1}^{+}$ & 5.531 & $7.22(9)$ \\
\hline \hline${ }^{135} \mathrm{La} \rightarrow{ }^{135} \mathrm{Ba}$ & & &
\end{tabular}

a $3 / 2,5 / 2^{+}$level at $1528 \mathrm{keV}$ in ${ }^{133} \mathrm{Ba}$.

b $3 / 2^{+}, 5 / 2^{+}$level at $676 \mathrm{keV}$ in ${ }^{133} \mathrm{Ba}$.

${ }^{\mathrm{c}} 3 / 2^{+}, 5 / 2^{+}, 7 / 2^{+}$level at $1112 \mathrm{keV}$ in ${ }^{133} \mathrm{Ba}$.

${ }^{\mathrm{d}} 3 / 2^{+}, 5 / 2^{+}$level at $980 \mathrm{keV}$ in ${ }^{135} \mathrm{Ba}$.

experimental one. Here, only the GT transition is involved. The reduced GT matrix element is found to be as small as 0.0061 , due to the cancellation of the many small components that make the matrix element. The largest contributions come from terms of the type $s_{v}^{\dagger} s_{\pi}\left[\left[\tilde{d}_{\nu} a_{\nu s_{1 / 2}}^{\dagger}\right]^{(3 / 2)} \tilde{a}_{\pi d_{5 / 2}}\right]^{(1)}$ and $s_{v}^{\dagger} s_{\pi}\left[\left[\tilde{d}_{\nu} a_{v s_{1 / 2}}^{\dagger}\right]^{(5 / 2)} \tilde{a}_{\pi d_{5 / 2}}\right]^{(1)}$. However, their coefficients are 0.0447 and -0.0334 , respectively. As a result, a small $B(\mathrm{GT})$ is obtained and this leads to a too large log $f t$ value as compared with the experiment. The $1 / 2_{1}^{+}$ground state IBFM wave function of the parent nucleus ${ }^{129} \mathrm{Ba}$ is mainly made of the $v s_{1 / 2}(36 \%)$ and $v d_{3 / 2}(47 \%)$ single-neutron configurations while, the $3 / 2_{1}^{+}$wave function corresponding to the daughter system ${ }^{129} \mathrm{Cs}$ is dominated by the $\pi g_{7 / 2}(41 \%)$ and $\pi d_{3 / 2}(31 \%)$ configurations. This difference in the parent and daughter states may partly account for the small GT strength.

\section{D. $\beta$ decays of odd- $A$ La nuclei}

From Tables V and VI, one sees that the computed $\log f t$ values for the $\beta^{+}$decays $\mathrm{La} \longrightarrow \mathrm{Ba}$ are larger than the ones obtained for the $\mathrm{Cs} \longleftrightarrow \mathrm{Xe}$ and $\mathrm{Ba} \longrightarrow \mathrm{Cs}$ decays (see Tables III and IV). However, the $\log f t$ values for some of the $\mathrm{La} \longrightarrow \mathrm{Ba}$ transitions are still too small as compared to the experimental ones [47]. This mainly occurs (see Table V) for the $\beta$ decay with $\Delta I=0$, where the $B(\mathrm{GT})$ as well as the $B(\mathrm{~F})$ transition strengths are too large. A typical example is the decay from the $5 / 2_{1}^{+}$ground state of ${ }^{135} \mathrm{La}$ to the $5 / 2_{1}^{+}$state of ${ }^{135} \mathrm{Ba}$. For this transition, the theoretical result underestimates the experimental $f t$ value by a factor of $\approx 10^{2}$ [see Table VI and Eq. (22)]. The dominant contribution to the GT transition strength comes from the term $s_{\nu}\left[a_{v d_{3 / 2}}^{\dagger} \tilde{a}_{\pi d_{5 / 2}}\right]^{(1)}$ with a coefficient in front of 0.414 . Here, the $\pi d_{5 / 2}$ and $v d_{3 / 2}$ configurations play the dominant role for the parent and daughter nuclei, respectively. The $5 / 2_{1}^{+}$wave function of ${ }^{135} \mathrm{La}$ is mainly based on the $\pi g_{7 / 2}$ configuration (89\%) while for ${ }^{135} \mathrm{Ba}$ the $5 / 2_{1}^{+}$excited state is mainly based on the $v d_{3 / 2}$ configuration (79\%) with a $v s_{1 / 2}$ component (19\%). Therefore, it is tempting to interpret the unexpectedly large $B(\mathrm{GT})$ value solely in terms of the relevant wave function contents. It must be kept in mind, however, that the $B(\mathrm{GT})$, as well as the $B(\mathrm{~F})$, transition strengths also depend upon the coefficients for the one-particle transfer operators [see Eqs. (8)-(11)] and those depend on other factors such as the occupation probabilities $v_{j}^{2}$ for the odd particle. In this particular example, the relevant coefficient is $\eta_{\nu 3 / 2, \pi 5 / 2} \theta_{\nu 3 / 2} \zeta_{\pi 5 / 2}$ [see Eqs. (12), (14), and (19)].

For those transitions, where the $B(\mathrm{GT})$ and $B(\mathrm{~F})$ values are found to be too small, i.e., the resulting $\log f t$ values are too large, cancellation seems to occur to a large extent between different components of the operators. For instance, for the transition ${ }^{135} \mathrm{La}\left(5 / 2_{1}^{+}\right) \longrightarrow{ }^{135} \mathrm{Ba}\left(3 / 2_{1}^{+}\right)$, the largest terms in the GT matrix element turn out to be $s_{\nu} s_{\pi}\left[a_{v d_{3 / 2}}^{\dagger}\left(d_{\pi}^{\dagger} \tilde{a}_{\pi g_{7 / 2}}\right)^{5 / 2}\right]^{(1)}, s_{\nu} s_{\pi}\left[a_{\nu d_{3 / 2}}^{\dagger}\left(d_{\pi}^{\dagger} \tilde{a}_{\pi d_{3 / 2}}\right)^{5 / 2}\right]^{(1)}$, and $s_{\nu}\left(a_{v d_{3 / 2}}^{\dagger} \tilde{a}_{\pi d_{5 / 2}}\right)^{(1)}$. The corresponding coefficients $(-0.0562$, -0.0246 , and 0.0979) almost cancel each other. The IBFM wave functions for the $5 / 2_{1}^{+}$and $3 / 2_{1}^{+}$states of ${ }^{135} \mathrm{La}$ and

${ }^{135} \mathrm{Ba}$ are mainly made of the $\pi g_{7 / 2}$ and $\nu d_{3 / 2}$ configurations. Once more, the $\log f t$ value for this transition is not completely accounted for by simply looking at the compositions of the wave functions. As we have already noted, the coefficients for the fermion transfer operators are determined by various factors such as the occupation probabilities $v_{j}^{2}$ and bosoncore wave functions. In turn, those factors are determined microscopically from the Gogny-D1M mean-field results.

\section{E. Sensitivity to the IBFM parameters}

As already mentioned in previous sections, the predicted $B(\mathrm{GT})$ and $B(\mathrm{~F})$ values appear to be somewhat sensitive to the details of the IBFM wave functions for the parent and daughter nuclei. In what follows, we study the dependence of the computed $\beta$-decay $\log f t$ values on the strength parameters of the IBFM.

As an example, let us consider the ${ }^{127} \mathrm{Cs} \longrightarrow{ }^{127} \mathrm{Xe}$ decay. We have performed additional IBFM calculations for ${ }^{127} \mathrm{Xe}$ with the strengths $\Gamma_{v}=1.6 \mathrm{MeV}, \Lambda_{v}=2.0 \mathrm{MeV}$, and $A_{v}=$ $-0.0 \mathrm{MeV}$. We will refer to this set of parameters as set $B$ while the original strengths, shown in Table II, will be denoted as set $A$. In Fig. 8, we have compared the energy spectra resulting from those two independent IBFM calculations with the 




FIG. 8. Level schemes for ${ }^{127} \mathrm{Xe}$. Results of two independent IBFM calculations, employing different sets of boson-fermion strengths, are compared with each other and with the experimental energy spectrum. The original strength parameters, shown in Table II, are denoted as set $A$ while the modified strengths are denoted as set $B$. For more details, see the main text.

experimental one. Both the set $B$ and set $A$ IBFM calculations reproduce the experimental excitation energies of states in the vicinity of the ground state with the same level of accuracy.

However, at higher excitation energy, the values obtained with set $B$ are much higher in energy than those with set $A$ mostly for levels with spin $I \geqslant 9 / 2$ ). The description of the corresponding $E 2$ and $M 1$ transitions and moments is worse with set $B$. However, the $\log f t$ values in the case of set $B$ are improved with respect to the ones obtained with set $A$. For instance, with set $B$ we have obtained $\log f t=4.486$ for the $1 / 2_{1}^{+} \rightarrow 1 / 2_{1}^{+}$transition from ${ }^{127} \mathrm{Cs}$ to ${ }^{127} \mathrm{Xe}$. This is closer to the experimental result $\log f t=6.53 \pm 0.06$ than the value $\log f t=3.711$ obtained with set $A$.

The difference in the predicted $\log f t$ values mainly comes from the corresponding $B(\mathrm{GT})$ rates. We have obtained the reduced GT matrix element $\left\langle 1 / 2_{1}^{+}\left\|\hat{\mathcal{O}}^{\mathrm{GT}}\right\| 1 / 2_{1}^{+}\right\rangle=-1.216$ with set $A$. In this case, the largest contribution comes from the term $s_{v}\left[a_{v s_{1 / 2}}^{\dagger} \tilde{a}_{\pi s_{1 / 2}}\right]^{(1)}$ with the coefficient of -0.837 . The contributions from the $v d_{3 / 2} \rightarrow \pi d_{5 / 2}$ and $v d_{5 / 2} \rightarrow \pi d_{5 / 2}$ terms are non-negligible but enter with opposite signs and therefore cancel each other. On the other hand, with set $B$, we have obtained $\left\langle 1 / 2_{1}^{+}\left\|\hat{\mathcal{O}}^{\mathrm{GT}}\right\| 1 / 2_{1}^{+}\right\rangle=0.448$ and the dominant component is the term $s_{v}\left[a_{v_{1 / 2}}^{\dagger} \tilde{a}_{\pi s_{1 / 2}}\right]^{(1)}$ with the coefficient 0.520 .

The $1 / 2_{1}^{+}$ground state for ${ }^{127} \mathrm{Xe}$ reads

$$
\begin{aligned}
\left|\frac{1^{+}}{{ }^{+}}\right\rangle= & \left(0.29\left|v s_{1 / 2}\right\rangle+0.55\left|v d_{3 / 2}\right\rangle\right. \\
& \left.+0.12\left|v d_{5 / 2}\right\rangle+0.04\left|v g_{7 / 2}\right\rangle\right) \otimes\left|{ }^{128} \mathrm{Xe}\right\rangle .
\end{aligned}
$$

with set $A$ while with set $B$ it takes the form

$$
\begin{aligned}
\left|\frac{1^{+}}{2}\right\rangle= & \left(0.62\left|v s_{1 / 2}\right\rangle+0.19\left|v d_{3 / 2}\right\rangle\right. \\
& \left.+0.16\left|v d_{5 / 2}\right\rangle+0.02\left|v g_{7 / 2}\right\rangle\right) \otimes\left|{ }^{128} \mathrm{Xe}\right\rangle .
\end{aligned}
$$

The two wave functions mainly differ in the amplitudes of the $v s_{1 / 2}$ and $v d_{3 / 2}$ configurations.

The $2 d_{3 / 2}$ single-particle orbital is the lowest in energy among the ones employed for ${ }^{127} \mathrm{Xe}$ (see Fig. 1 of Ref. [34]). Of the three boson-fermion terms, the exchange term is particularly important in mixing different single-particle configurations and the most significant difference, between the parameters of set $A$ and set $B$ is perhaps the larger exchange strength $\Lambda_{v}$ used for set $B$. Because of this, the mixing of the $v s_{1 / 2}$ single-particle components into the $1 / 2_{1}^{+}$ground state of

${ }^{127} \mathrm{Xe}$ is stronger. On the other hand, the $1 / 2_{1}^{+}$ground state of the parent nucleus ${ }^{127} \mathrm{Cs}$ can be decomposed as follows:

$$
\begin{aligned}
\left|\frac{1}{2}_{1}^{+}\right\rangle= & \left(0.24\left|\pi s_{1 / 2}\right\rangle+0.08\left|\pi d_{3 / 2}\right\rangle\right. \\
& \left.+0.66\left|\pi d_{5 / 2}\right\rangle+0.02\left|\pi g_{7 / 2}\right\rangle\right) \otimes\left|{ }^{126} \mathrm{Xe}\right\rangle .
\end{aligned}
$$

which has a similar mixing amplitude for the $s_{1 / 2}$ singleproton configuration to the $1 / 2_{1}^{+}$wave function for the daughter nucleus.

\section{SUMMARY AND CONCLUDING REMARKS}

In this paper, we have presented a consistent description of the low-energy excitation spectra and $\beta$ decay of odd- $A$ nuclei within the IBFM based on input from realistic mean field calculations. The $(\beta, \gamma)$ potential energy surfaces for eveneven nuclei, the spherical single-particle energies, and the occupation probabilities for the neighboring odd- $A$ nuclei have been computed microscopically within the constrained HFB scheme based on the Gogny-D1M EDF. Those quantities are used as microscopic input to access the spectroscopic properties in odd- $A$ nuclei within the IBFM. Only the three coupling constants for the boson-fermion interaction terms have been fitted to experimental data as to reproduce reasonably well the low-lying energy levels in each of the studied odd- $A$ systems. Having the IBFM wave functions for the parent and daughter nuclei, the GT and F transition strengths have been computed without any additional phenomenological parameter.

The low-lying, positive-parity excitation spectra are reproduced reasonably well for the even-even $\mathrm{Xe}$ and $\mathrm{Ba}$, the neighboring odd- $N$ Xe and $\mathrm{Ba}$ as well as the odd- $Z$ Cs and La nuclei. The isotopic dependence of the energy levels in the studied odd- $A$ nuclei, and in the corresponding even-even Xe and $\mathrm{Ba}$ cores, points to an evolution from prolate to $\gamma$-soft and to nearly spherical shapes. Electromagnetic properties, such as the $B(E 2)$ transition rates and magnetic dipole moments, are well reproduced. We have obtained $\beta$-decay $\log f t$ values that are systematically smaller than the experimental ones. Those $\log f t$ values mainly depend on the details of the IBFM wave functions for the parent and daughter nuclei and, in many cases, they lead to unexpectedly large $B(\mathrm{GT})$ and/or $B(\mathrm{~F})$ values. The same problem has been often observed in other theoretical approaches suggesting the necessity of a quenching factor for $g_{A}$. On the other hand, we do not introduce such a quenching in the present study. The results for the $\beta$-decay $\log f t$ values serve as a sensitive test for the employed theoretical method and may indicate certain improvements of the method, including the descriptions of 
the low-lying structures for both the even-even core and odd-mass parent and daughter nuclei. Possible ways to do so are the inclusions of new terms in the IBM and IBFM Hamiltonians or new degrees of freedom in the IBFM such as an intruder orbital coming from a next major shell. Another possibility to improve the description of the $\log f t$ values is to incorporate the effects of higher order terms in the one-particle transfer operators in Eqs. (8)-(11), as examined in Ref. [24]. This paper presents an implementation of the EDF-based IBFM approach in the description of $\beta$ decay and, therefore, those extensions of the method are beyond the scope the present work and will be explored in the near future.

In spite of the reduced number of empirical parameters in the model, it is possible to describe the detailed excitation spectra for even-even, odd- $A$, and odd-odd nuclear systems, and $\beta$-decay properties simultaneously and with reasonable computational time. Keeping all this in mind, we conclude that our description of the low-lying states and $\beta$-decay properties of odd- $A$ nuclei within the EDF-based IBFM approach is fairly promising in the study of fundamental nuclear processes.

\section{ACKNOWLEDGMENTS}

We thank $\mathrm{N}$. Yoshida for helping us with the $\beta$-decay calculations The work of K.N. is financed within the Tenure Track Pilot Programme of the Croatian Science Foundation and the École Polytechnique Fédérale de Lausanne, and the Project TTP-2018-07-3554 Exotic Nuclear Structure and Dynamics, with funds of the Croatian-Swiss Research Programme. The work of L.M.R. was supported by Spanish Ministry of Economy and Competitiveness (MINECO) Grant No. PGC2018-094583-B-I00.
[1] I. Dillmann, K.-L. Kratz, A. Wöhr, O. Arndt, B. A. Brown, P. Hoff, M. Hjorth-Jensen, U. Köster, A. N. Ostrowski, B. Pfeiffer et al. (the ISOLDE Collaboration), Phys. Rev. Lett. 91, 162503 (2003).

[2] S. Nishimura, Z. Li, H. Watanabe, K. Yoshinaga, T. Sumikama, T. Tachibana, K. Yamaguchi, M. Kurata-Nishimura, G. Lorusso, Y. Miyashita et al., Phys. Rev. Lett. 106, 052502 (2011).

[3] M. Quinn, A. Aprahamian, J. Pereira, R. Surman, O. Arndt, T. Baumann, A. Becerril, T. Elliot, A. Estrade, D. Galaviz et al., Phys. Rev. C 85, 035807 (2012).

[4] G. Lorusso, S. Nishimura, Z. Y. Xu, A. Jungclaus, Y. Shimizu, G. S. Simpson, P.-A. Söderström, H. Watanabe, F. Browne, P. Doornenbal et al., Phys. Rev. Lett. 114, 192501 (2015).

[5] R. Caballero-Folch, C. Domingo-Pardo, J. Agramunt, A. Algora, F. Ameil, A. Arcones, Y. Ayyad, J. Benlliure, I. N. Borzov, M. Bowry et al., Phys. Rev. Lett. 117, 012501 (2016).

[6] K. Langanke and G. Martínez-Pinedo, Rev. Mod. Phys. 75, 819 (2003).

[7] J. T. Suhonen, Frontiers Phys. 5, 55 (2017).

[8] P. Sarriguren, E. M. de Guerra, and A. Escuderos, Nucl. Phys. A 691, 631 (2001).

[9] F. Šimkovic, V. Rodin, A. Faessler, and P. Vogel, Phys. Rev. C 87, 045501 (2013).

[10] P. Pirinen and J. Suhonen, Phys. Rev. C 91, 054309 (2015).

[11] T. Marketin, L. Huther, and G. Martínez-Pinedo, Phys. Rev. C 93, 025805 (2016).

[12] M. T. Mustonen and J. Engel, Phys. Rev. C 93, 014304 (2016).

[13] J.-U. Nabi and M. Böyükata, Nucl. Phys. A 947, 182 (2016).

[14] E. Caurier, G. Martínez-Pinedo, F. Nowacki, A. Poves, and A. P. Zuker, Rev. Mod. Phys. 77, 427 (2005).

[15] M. Honma, T. Otsuka, T. Mizusaki, M. Hjorth-Jensen, and B. A. Brown, J. Phys.: Conf. Ser. 20, 7 (2005).

[16] S. Yoshida, Y. Utsuno, N. Shimizu, and T. Otsuka, Phys. Rev. C 97, 054321 (2018).

[17] F. Dellagiacoma, Ph.D. thesis, Yale University, New Haven, CT, 1988.

[18] P. Navrátil and J. Dobes, Phys. Rev. C 37, 2126 (1988).

[19] F. Iachello and P. Van Isacker, The Interacting Boson-Fermion Model (Cambridge University Press, Cambridge, UK, 1991).

[20] N. Yoshida, L. Zuffi, and S. Brant, Phys. Rev. C 66, 014306 (2002).
[21] L. Zuffi, S. Brant, and N. Yoshida, Phys. Rev. C 68, 034308 (2003)

[22] S. Brant, N. Yoshida, and L. Zuffi, Phys. Rev. C 70, 054301 (2004).

[23] S. Brant, N. Yoshida, and L. Zuffi, Phys. Rev. C 74, 024303 (2006).

[24] E. Mardones, J. Barea, C. E. Alonso, and J. M. Arias, Phys. Rev. C 93, 034332 (2016).

[25] P. Ring and P. Schuck, The Nuclear Many-Body Problem (Springer-Verlag, Berlin, 1980).

[26] M. Bender, P.-H. Heenen, and P.-G. Reinhard, Rev. Mod. Phys. 75, 121 (2003).

[27] L. M. Robledo, T. R. Rodríguez, and R. R. Rodríguez-Guzmán, J. Phys. G: Nucl. Part. Phys. 46, 013001 (2019).

[28] B. Bally, B. Avez, M. Bender, and P.-H. Heenen, Phys. Rev. Lett. 113, 162501 (2014).

[29] M. Borrajo and J. L. Egido, Eur. Phys. J. A 52, 277 (2016).

[30] K. Nomura, T. Nikšić, and D. Vretenar, Phys. Rev. C 93, 054305 (2016).

[31] K. Nomura, R. Rodríguez-Guzmán, and L. M. Robledo, Phys. Rev. C 96, 014314 (2017).

[32] F. Iachello and O. Scholten, Phys. Rev. Lett. 43, 679 (1979).

[33] K. Nomura, R. Rodríguez-Guzmán, and L. M. Robledo, Phys. Rev. C 99, 034308 (2019).

[34] K. Nomura, R. Rodríguez-Guzmán, and L. M. Robledo, Phys. Rev. C 96, 064316 (2017).

[35] F. Iachello and A. Arima, The Interacting Boson Model (Cambridge University Press, Cambridge, MA, 1987).

[36] J. Arias, C. Alonso, and R. Bijker, Nucl. Phys. A 445, 333 (1985).

[37] S. Goriely, S. Hilaire, M. Girod, and S. Péru, Phys. Rev. Lett. 102, 242501 (2009).

[38] J. Decharge, M. Girod, and D. Gogny, Phys. Lett. B 55, 361 (1975).

[39] K. Nomura, R. Rodríguez-Guzmán, and L. M. Robledo, Phys. Rev. C 101, 014306 (2020).

[40] T. Otsuka, A. Arima, and F. Iachello, Nucl. Phys. A 309, 1 (1978).

[41] O. Scholten, Prog. Part. Nucl. Phys. 14, 189 (1985).

[42] J. M. Arias, C. E. Alonso, and M. Lozano, Phys. Rev. C 33, 1482 (1986). 
[43] J. N. Ginocchio and M. W. Kirson, Nucl. Phys. A 350, 31 (1980).

[44] K. Nomura, N. Shimizu, and T. Otsuka, Phys. Rev. Lett. 101, 142501 (2008).

[45] K. Nomura, N. Shimizu, and T. Otsuka, Phys. Rev. C 81, 044307 (2010).
[46] J. Beringer, J. F. Arguin, R. M. Barnett, K. Copic, O. Dahl, D. E. Groom, C. J. Lin, J. Lys, H. Murayama, C. G. Wohl et al. (Particle Data Group), Phys. Rev. D 86, 010001 (2012).

[47] Brookhaven National Nuclear Data Center, http://www.nndc. bnl.gov. 